\title{
FAMILIES OF ARTINIAN AND LOW DIMENSIONAL DETERMINANTAL RINGS.
}

\author{
JAN O. KLEPPE
}

\begin{abstract}
Let $\operatorname{GradAlg}(H)$ be the scheme parameterizing graded quotients of $R=$ $k\left[x_{0}, \ldots, x_{n}\right]$ with Hilbert function $H$ (it is a subscheme of the Hilbert scheme of $\mathbb{P}^{n}$ if we restrict to quotients of positive dimension, see definition below). A graded quotient $A=R / I$ of codimension $c$ is called standard determinantal if the ideal $I$ can be generated by the $t \times t$ minors of a homogeneous $t \times(t+c-1)$ matrix $\left(f_{i j}\right)$. Given integers $a_{0} \leq a_{1} \leq \ldots \leq a_{t+c-2}$ and $b_{1} \leq \ldots \leq b_{t}$, we denote by $W_{s}(\underline{b} ; \underline{a}) \subset \operatorname{GradAlg}(H)$ the stratum of determinantal rings where $f_{i j} \in R$ are homogeneous of degrees $a_{j}-b_{i}$.

In this paper we extend previous results on the dimension and codimension of $W_{s}(\underline{b} ; \underline{a})$ in $\operatorname{GradAlg}(H)$ to artinian determinantal rings, and we show that $\operatorname{GradAlg}(H)$ is generically smooth along $W_{s}(\underline{b} ; \underline{a})$ under some assumptions. For zero and one dimensional determinantal schemes we generalize earlier results on these questions. As a consequence we get that the general element of a component $W$ of the Hilbert scheme of $\mathbb{P}^{n}$ is glicci provided $W$ contains a standard determinantal scheme satisfying some conditions. We also show how certain ghost terms disappear under deformation while other ghost terms remain and are present in the minimal resolution of a general element of $\operatorname{GradAlg}(H)$.
\end{abstract}

Keywords. Determinantal rings, Deformations, Hilbert schemes, Artinian rings.

AMS Subject Classification. 13C40, 14C05, 13D10 (Primary), 14M12, 13E10, 13D02, 13D07, 13A02, 13H10 (Secondary).

\section{INTRODUCTION}

The main goal of this paper is to generalize previous results on maximal families of determinantal schemes, notable to cover maximal families of artinian determinantal $k$ algebras. Recall that a $k$-algebra $A \simeq R / I, R=k\left[x_{0}, \ldots, x_{n}\right]$, of codimension $c$ is called determinantal if the ideal $I$ can be generated by the $r \times r$ minors of a homogeneous $p \times q$ matrix $\left(f_{i j}\right)$ with $c=(p-r+1)(q-r+1)$. A is called standard (resp. good) determinantal. if $r=\min (p, q)$ (resp. $r=\min (p, q)$ and $A$ is a generic complete intersection of $R$ ).

Let $\operatorname{GradAlg}(H)$ be the "Hilbert scheme of constant Hilbert function", i.e. the scheme parameterizing graded quotients $A$ of $R$ of $\operatorname{depth} A \geq \min (1, \operatorname{dim} A)$ and with Hilbert function $H$. Given integers $a_{0} \leq a_{1} \leq \ldots \leq a_{t+c-2}$ and $b_{1} \leq \ldots \leq b_{t}$, we denote by $W(\underline{b} ; \underline{a})\left(\operatorname{resp} . W_{s}(\underline{b} ; \underline{a})\right)$ the stratum in $\operatorname{Grad} \operatorname{Alg}(H)$ consisting of good (resp. standard) determinantal $k$-algebras where $f_{i j}$ are homogeneous polynomials of degree $a_{j}-b_{i}$. Then $W_{s}(\underline{b} ; \underline{a})$ is irreducible, $W_{s}(\underline{b} ; \underline{a}) \neq \emptyset$ if $a_{i-1}>b_{i}$ for $1 \leq i \leq t$, and the closures $\overline{W_{s}(\underline{b} ; \underline{a})}$ and $\overline{W(\underline{b} ; \underline{a})}$ are equal if $n \geq c$ (Proposition 2.1).

In this paper we focus on the following problems.

(1) Determine when $\overline{W_{s}(\underline{b} ; \underline{a})}$ is an irreducible component of $\operatorname{GradAlg}(H)$.

(2) Find the codimension of $W_{s}(\underline{b} ; \underline{a})$ in $\operatorname{Grad} \operatorname{Alg}(H)$ if its closure is not a component. 
(3) Determine when $\operatorname{GradAlg}(H)$ is generically smooth along $W_{s}(\underline{b} ; \underline{a})$.

These questions have been considered in several papers, and in 31 we solve all these problems completely provided $n-c>1$ and $a_{0}>b_{t}$. In this case the closure of $W_{s}(\underline{b} ; \underline{a})$ is a generically smooth irreducible component of the usual Hilbert scheme $\operatorname{Hilb}\left(\mathbb{P}^{n}\right)$, as well as of $\operatorname{GradAlg}(H)$ (see Theorem 4.6), i.e. [34, Conjecture 4.2] holds, and $\operatorname{dim} W_{s}(\underline{b} ; \underline{a})$ is determined (equal to $\lambda$ in Theorem 4.1, whence [34, Conjecture 4.1] holds for $n-c>0$ ), see also [13] for a somewhat different approach to these problems. So we only need to consider the case $n+1-c \in\{0,1,2\}$, and in [29] we solve problems (1)-(3) for determinantal schemes with $n+1-c \in\{1,2\}$ under certain assumptions. In this paper we concentrate on artinian determinantal $k$-algebras $(n+1-c=0)$, and we prove the main Theorems 4.1 and 4.6 under conditions that allow $c$ to be $n+1$. In addition we prove a new result (Theorem 4.8, extending Theorem 4.6) which applies when $\operatorname{dim} W_{s}(\underline{b} ; \underline{a}) \neq \lambda$. This theorem implies that the general element of an irreducible component $W$ of the Hilbert scheme of $\mathbb{P}^{n}$ is glicci (in the Gorenstein liaison class of a complete intersection) provided $W$ contains a standard determinantal scheme satisfying some conditions (Corollary 4.14). For an introduction to glicciness, see [32], and see [11] and its references for further developments. Finally, in Sec. 6, we generalize and complete several results of [29] for families of determinantal schemes of dimension 0 or 1 , some of which occurred in [32, 33], and we slightly extend a result of 34 .

We have used two different strategies to attack the problems (1) to (3). Indeed in [32, 33, 29, 34] we successively deleted columns of the $t \times(t+c-1)$ matrix $\mathcal{A}$ associated to a determinantal scheme $X:=\operatorname{Proj}(A)$ to get a nest ("flag") of closed subschemes $X=X_{c} \subset X_{c-1} \subset \ldots \subset X_{2} \subset \mathbb{P}^{n}$ and we proved our results inductively by considering the smoothness of the Hilbert flag scheme of pairs and its natural projections to Hilbert schemes. On the other hand, in [31] we compared deformations of $A$ with deformation of the cokernel $M$ of the map $\oplus_{j=0}^{t+c-2} R\left(-a_{j}\right) \rightarrow \oplus_{i=1}^{t} R\left(-b_{i}\right)$ induced by $\mathcal{A}$. This latter approach turned out to be quite successful, and it indeed solved problem (1)-(3) for $n-c>1$. It is this approach that we generalize to the artinian case, only introducing an extra assumption $\left({ }_{0} \operatorname{Hom}_{A}(M, M) \simeq k\right)$ in the theorems. In fact we show that the main results of [31] hold, whence partially solving problems (1) to (3) also for $n=c-1$.

For $c=2$ all assumptions of the theorems are fulfilled. We even replace $R$ by any CohenMacaulay quotient $\bar{R}$ of $R$ and solve the problems above for determinantal quotients of codimension $c=2$ of $\bar{R}$ (Theorem 4.15). For $c>2$ we need to verify that certain Extigroups vanish to apply our results when $n+1-c \in\{0,1,2\}$. In this paper we accomplish this by using Macaulay 2 ([15]). We give many examples, supported by Macaulay 2 computations, to illustrate the theorems in the artinian case.

In Sec. 5 we consider ghost terms, i.e. common free summands in consecutive terms of the minimal $R$-free resolution of $A$, and we show that some of them disappear, others remain unchanged under suitable generizations (deformations to a more general algebra) in $\operatorname{GradAlg}(H)$. If $A$ is general in $\operatorname{GradAlg}(H)$ and the assumptions of Theorem 4.8 are fulfilled (e.g. $\operatorname{dim} A \geq 3$ and $a_{0} \geq b_{t}$ ), one may easily describe all ghost terms in its minimal free resolution while it seems hard to get improved results when $\operatorname{dim} A \leq 2$ (Proposition 5.1, Remark 5.3), although examples indicate a possible connection (Remark 4.12). 
In the proofs we use the exactness of the Buchsbaum-Rim complex ([6, 10 $)$ and the 5term exact sequence associated to the spectral sequence $E_{2}^{p, q}:=\operatorname{Ext}_{A}^{p}\left(\operatorname{Tor}_{q}^{R}(A, M), M\right) \Rightarrow$ $\operatorname{Ext}_{R}^{p+q}(M, M)$ which, in view of Fitting's lemma, plays a surprisingly important role in comparing the deformation functors of $M$ and $A$.

Notation: Throughout $\mathbb{P}:=\mathbb{P}^{n}$ is the projective $n$-space over an algebraically closed field $k$ (except in Theorem 4.15 where we allow $k \neq \bar{k}$ ), $R=k\left[x_{0}, x_{1}, \ldots, x_{n}\right]$ is a polynomial ring and $\mathfrak{m}=\left(x_{0}, \ldots, x_{n}\right)$. If $X \subset Y$ are closed subschemes of $\mathbb{P}^{n}$, we denote by $\mathcal{I}_{X / Y}$ (resp. $\mathcal{N}_{X / Y}$ ) the ideal (resp. normal) sheaf of $X$ in $Y$, and we omit $/ Y$ if $Y=\mathbb{P}^{n}$. Let $I_{X}=H_{*}^{0}\left(\mathcal{I}_{X}\right) \subset R$ be the saturated homogeneous ideal of $X \subset \mathbb{P}^{n}$. When we write $X=\operatorname{Proj}(A)$ we always let $A:=R / I_{X}$ and $K_{A}=\operatorname{Ext}_{R}^{c}(A, R)(-n-1)$ be the canonical module of $A$ or $X$ where $c=\operatorname{codim}_{\mathbb{P}} X:=n-\operatorname{dim} X$. Note that by the codimension, $\operatorname{codim}_{Y} X$, of an irreducible $X$ in a not necessarily equidimensional scheme $Y$ we mean $\operatorname{dim} \mathcal{O}_{Y, x}-\operatorname{dim} X$, where $x$ is a general $k$-point of $X$. We let ${ }_{v} \operatorname{Ext}(F, G)$ be the degree- $v$ part of the graded module $\operatorname{Ext}(F, G)$. Moreover we denote by $\operatorname{hom}(\mathcal{F}, \mathcal{G})=\operatorname{dim}_{k} \operatorname{Hom}(\mathcal{F}, \mathcal{G})$ the dimension of the group of morphisms between coherent $\mathcal{O}_{X}$-modules and we use small letters for the $k$-vector space dimension of similar groups.

\section{Preliminaries}

2.1. Hilbert schemes and Hilbert function strata. We denote the Hilbert scheme with the Hilbert polynomial $p \in \mathbb{Q}[s]$ by $\operatorname{Hilb}^{p}\left(\mathbb{P}^{n}\right)$, cf. [16] for existence and [39] for the local theory. Similarly $\operatorname{GradAlg}^{H}(R)$, or $\operatorname{Hilb}^{H}\left(\mathbb{P}^{n}\right)$ when $\operatorname{dim} A>0$, is the representing object of the functor that parameterizes flat families of graded quotients $A$ of $R$ of $\operatorname{depth}_{\mathfrak{m}} A \geq \min (1, \operatorname{dim} A)$ and with Hilbert function $H, H(i)=\operatorname{dim} A_{i}([26$, [27, 17]). We allow calling it "the postulation Hilbert scheme" $([28, \S 1.1])$ even though it may be different from the parameter space studied by Gotzmann and Iarrobino ([14, 21]) who study the "same" scheme with the reduced scheme structure. We let $(A)$, or $(X)$ where $X=\operatorname{Proj}(A)$, denote the point of $\operatorname{GradAlg}(H):=\operatorname{GradAlg}^{H}(R)$ that corresponds to $A$. Note that if $\operatorname{depth}_{\mathfrak{m}} A \geq 1$ and ${ }_{0} \operatorname{Hom}_{R}\left(I_{X}, H_{\mathfrak{m}}^{1}(A)\right)=0$, then

$$
\operatorname{Grad} \operatorname{Alg}(H) \simeq \operatorname{Hilb}^{p}\left(\mathbb{P}^{n}\right) \text { at }(X),
$$

and hence we have an isomorphism ${ }_{0} \operatorname{Hom}\left(I_{X}, A\right) \simeq H^{0}\left(\mathcal{N}_{X}\right)$ of their tangent spaces (cf. [12] for the case $\operatorname{depth}_{\mathfrak{m}} A \geq 2$, and (9) of [27] for the general case). If $X$ is generically a complete intersection, then ${ }_{0} \operatorname{Ext}_{A}^{1}\left(I_{X} / I_{X}^{2}, A\right)$ is an obstruction space of $\operatorname{GradAlg}(H)$ at $(A)$, and hence of $\operatorname{Hilb}^{p}\left(\mathbb{P}^{n}\right)$ at $(X)$ if (2.1) holds. Indeed for any quotient $A=R / I$, we may define $\mathrm{H}_{2}(R, A, A):=\operatorname{ker}\left(S_{2}(I) \rightarrow I^{2}\right)$ where $S_{2}(I)$ is the $2^{\text {nd }}$ symmetric power of $I$, cf. [1], p.106. Then $\operatorname{GradAlg}(H)$ is smooth at $(A)$ if

$$
{ }_{0} \operatorname{Ext}_{A}^{1}\left(I / I^{2}, A\right)=0 \text { and }{ }_{0} \operatorname{Hom}_{A}\left(\mathrm{H}_{2}(R, A, A), A\right)=0,
$$

and observe that the latter group vanishes if $\mathrm{A}$ is generically a complete intersection of $R$ [27, $\S 1.1]$. By definition $X$ (resp. A) is called unobstructed if $\operatorname{Hilb}^{p}\left(\mathbb{P}^{n}\right.$ ) (resp. $\operatorname{GradAlg}(H))$ is smooth at $(X)(\operatorname{resp} .(A))$.

We say that $X$ is general in some irreducible subset $W \subset \operatorname{Hilb}^{p}\left(\mathbb{P}^{n}\right)$ if $(X)$ belongs to a sufficiently small open subset $U$ of $W$ such that any $(X)$ in $U$ has all the openness properties that we want to require. We define " $A$ general in $\operatorname{GradAlg}(H)$ " similarly. 
2.2. Determinantal rings and schemes. We mainly maintain the notions and notations from [29, 31], but we need to extend some results to artinian determinantal $k$-algebras. For a more general background of determinantal rings and schemes, see [4, 10, 3, 44.

Indeed let

$$
\varphi: F=\bigoplus_{i=1}^{t} R\left(b_{i}\right) \longrightarrow G:=\bigoplus_{j=0}^{t+c-2} R\left(a_{j}\right)
$$

be a graded morphism of free $R$-modules, let $\mathcal{A}=\left(f_{i j}\right)_{i=1, \ldots, t}^{j=0, \ldots, t+2}, \operatorname{deg} f_{i j}=a_{j}-b_{i}$, be a matrix which represents the dual $\varphi^{*}:=\operatorname{Hom}_{R}(\varphi, R)$ and let $I(\mathcal{A}):=I_{t}(\mathcal{A})$ be the ideal of $R$ generated by the maximal minors of $\mathcal{A}$. We always suppose $t \geq 2$ (unless explicitly allowing $t=1$ ) and

$$
c \geq 2, \quad b_{1} \leq \ldots \leq b_{t} \quad \text { and } \quad a_{0} \leq a_{1} \leq \ldots \leq a_{t+c-2} .
$$

A codimension $c$ quotient $A=R / I$ (resp. subscheme $X \subset \mathbb{P}^{n}$ if $n \geq c$ ) is called standard determinantal if $I:=I(\mathcal{A})\left(\operatorname{resp} . I_{X}:=I(\mathcal{A})\right)$ for some homogeneous $t \times(t+c-1)$ matrix $\mathcal{A}$ as above. They are good determinantal if additionally, the ideal $I_{t-1}(\mathcal{A})$ of submaximal minors has at least codimension $c+1$ in $R$, i.e. $R / I(\mathcal{A})$ is a generic complete intersection of $R$. If $t=1$, standard, as well as good determinantal are complete intersections of $R$.

Given integers $b_{i}$ and $a_{j}$ satisfying (2.4) we let $W_{s}(\underline{b} ; \underline{a} ; R)(\operatorname{resp} . W(\underline{b} ; \underline{a} ; R))$ be the stratum in $\operatorname{GradAlg}(H):=\operatorname{GradAlg}^{H}(R)$ consisting of standard (resp. good) determinantal quotients, cf. (2.1), and we usually omit $R$ in this notation. Note that we do not require $\mathcal{A}$ to be minimal (i.e. $f_{i j}=0$ when $b_{i}=a_{j}$ ) for $R / I_{t}(\mathcal{A})$ to belong to $W_{s}(\underline{b} ; \underline{a}):=W_{s}(\underline{b} ; \underline{a} ; R)$. In examples, however, we mainly consider determinantal rings with positive degree matrix (i.e. for every $i, j, b_{i}<a_{j}$, and recall that $a_{j}-b_{i}=1$ is called the linear case).

Proposition 2.1. The closure of $W_{s}(\underline{b} ; \underline{a})$ in $\operatorname{GradAlg}(H)$ is irreducible, and

$$
W_{s}(\underline{b} ; \underline{a}) \neq \emptyset \quad \Leftrightarrow \quad a_{i-1} \geq b_{i} \text { for all } i \text { and } a_{i-1}>b_{i} \text { for some } i \text {. }
$$

Moreover if $n-c \geq 0$, then $\overline{W_{s}(\underline{b} ; \underline{a})}=\overline{W(\underline{b} ; \underline{a})}$. And if $n-c=-1$, then every $A=R / I$ of $W(\underline{b} ; \underline{a})$ satisfies $I_{t-1}(\mathcal{A})=R$, i.e. is a complete intersection (c.i.) of $R$.

Proof. For $W_{s}(\underline{b} ; \underline{a})$ to be non-empty we refer to $(2.2)$ of [34] whose arguments carry over to the artinian case. The text after $[34,(2.2)]$ shows also that $\overline{W_{s}(\underline{b} ; \underline{a})}=\overline{W(\underline{b} ; \underline{a})}$ and that this locus is irreducible in the non-artinian case $(n \geq c)$. To see that $W_{s}(\underline{b} ; \underline{a})$ is irreducible also when $W_{s}(\underline{b} ; \underline{a})$ parametrizes artinian $k$-algebras we consider the affine scheme $\mathbb{V}=\operatorname{Hom}_{R}\left(G^{*}, F^{*}\right)$ whose rational points correspond to $t \times(t+c-1)$ matrices. Since the vanishing of $\operatorname{Ext}_{R}^{i}\left(R / I_{t}(\mathcal{A}), R\right)$ is an open property, the subset $U$ of $\mathbb{V}$ of matrices such that $I_{t}(\mathcal{A})$ has maximal codimension in $R$ is open and irreducible. Then, since there is an obvious morphism from $U$ onto $W_{s}(\underline{b} ; \underline{a})$, it follows that $\overline{W_{s}(\underline{b} ; \underline{a})}$ is irreducible.

Finally, for $A$ artinian and good determinantal, $I_{t-1}(\mathcal{A})$ is not contained in $\mathfrak{m}$ because this would imply $\operatorname{dim} R / I_{t-1}(\mathcal{A})=\operatorname{dim} R / I_{t}(\mathcal{A})=0$. Thus $I_{t-1}(\mathcal{A})=R$, and it follows that $A$ is a c.i. of $R$ by Corollary 5.2 .

Remark 2.2. In earlier papers on determinantal rings we often assumed that $A$ was good determinantal. By [33, Lem. 3.2] good determinantal rings satisfy $A \simeq \operatorname{Hom}_{A}(M, M)$ 
where $M:=\operatorname{coker} \varphi^{*}$. This and the fact that they are generic complete intersections of $R$, was often used in the proofs of the deformation results in [32, 33, 29, 31]. To generalize earlier results to the artinian case, we see from Proposition 2.1 that we can not assume $A$ good determinantal any more. Also $A \simeq \operatorname{Hom}_{A}(M, M)$ has to be dropped because this seems to never hold for $A$ artinian. It turns, however, out that only assuming ${ }_{0} \operatorname{Hom}_{A}(M, M) \simeq k$ and $A$ standard determinantal often allows us to generalize the proofs.

In the following let $A=R / I_{t}(\mathcal{A})$ be standard determinantal, i.e. $(A) \in W_{s}(\underline{b} ; \underline{a})$ and let $X=\operatorname{Proj}(A)$ if $\operatorname{dim} A>0$. Then $R$-free resolutions of $A$ and $M:=M_{\mathcal{A}}:=\operatorname{coker} \varphi^{*}$ are given by the Eagon-Northcott and Buchsbaum-Rim complexes respectively, see [6, 9, 10]. These resolutions are minimal if $\mathcal{A}$ is minimal. By e.g. [31, (2.3), (2.4)] the resolutions are

$$
\begin{array}{r}
0 \longrightarrow \wedge^{t+c-1} G^{*} \otimes S_{c-1}(F) \otimes \wedge^{t} F \longrightarrow \cdots \rightarrow \wedge^{t+i} G^{*} \otimes S_{i}(F) \otimes \wedge^{t} F \\
\rightarrow \ldots \rightarrow \wedge^{t} G^{*} \otimes S_{0}(F) \otimes \wedge^{t} F \longrightarrow R \longrightarrow A \longrightarrow 0, \quad \text { and } \\
0 \rightarrow \wedge^{t+c-1} G^{*} \otimes S_{c-2}(F) \otimes \wedge^{t} F \rightarrow \cdots \rightarrow \wedge^{t+i+1} G^{*} \otimes S_{i}(F) \otimes \wedge^{t} F \\
\rightarrow \ldots \rightarrow \wedge^{t+1} G^{*} \otimes S_{0}(F) \otimes \wedge^{t} F \rightarrow G^{*} \stackrel{\varphi^{*}}{\rightarrow} F^{*} \rightarrow M \rightarrow 0 .
\end{array}
$$

The matrix $\mathcal{A}_{i}$ obtained by deleting the last $c-i$ columns defines a morphism

$$
\varphi_{i}: F=\bigoplus_{i=1}^{t} R\left(b_{i}\right) \longrightarrow G_{i}:=\bigoplus_{j=0}^{t+i-2} R\left(a_{j}\right) .
$$

and we suppose that all entries of the deleted columns belong to $\mathfrak{m}$. Then the $k$-algebra $D_{i} \cong R / I_{D_{i}}$ given by the maximal minors of $\mathcal{A}_{i}$ is standard determinantal by [2] and $\varphi_{i}$ is injective. Letting $B_{i}=\operatorname{coker} \varphi_{i}$ and $M_{i}=\operatorname{coker} \varphi_{i}^{*}$, we have an exact sequence

$$
0 \rightarrow B_{i}^{*} \rightarrow G_{i}^{*} \stackrel{\varphi_{i}^{*}}{\longrightarrow} F^{*} \longrightarrow M_{i} \cong \operatorname{Ext}_{R}^{1}\left(B_{i}, R\right) \longrightarrow 0 .
$$

Then $M_{i}$ is a $D_{i}$-module, $R \rightarrow D_{i} \rightarrow D_{i+1} \ldots \rightarrow D_{c}=A$, and there is an exact sequence

$$
0 \longrightarrow D_{i} \longrightarrow M_{i}\left(a_{t+i-1}\right) \longrightarrow M_{i+1}\left(a_{t+i-1}\right) \longrightarrow 0
$$

in which $D_{i} \longrightarrow M_{i}\left(a_{t+i-1}\right)$ is a regular section that defines $D_{i+1}$ (also when $i+1=c$ and $D_{c}$ is artinian [36, Lem. 3.6]). Hence the sequence

$$
0 \longrightarrow I_{D_{i+1} / D_{i}} \simeq \operatorname{Hom}_{D_{i}}\left(M_{i}\left(a_{t+i-1}\right), D_{i}\right) \longrightarrow D_{i} \longrightarrow D_{i+1} \longrightarrow 0 \text {. }
$$

is exact. By (2.6) $M_{i}$ is a maximal Cohen-Macaulay $D_{i}$-module and so is $I_{D_{i+1} / D_{i}}$ by (2.10). Moreover by e.g. (2.6),$K_{D_{i}}(n+1) \cong S_{i-1} M_{i}\left(\ell_{i}\right)$ where $\ell_{i}:=\sum_{j=0}^{t+i-2} a_{j}-\sum_{q=1}^{t} b_{q}$.

Lemma 2.3. With the above notation (and $M=M_{c}$ ), there are exact sequences

$$
\begin{aligned}
& 0 \rightarrow \operatorname{Hom}_{R}\left(M_{i}, M\right) \rightarrow F \otimes_{R} M \rightarrow G_{i} \otimes_{R} M \rightarrow B_{i} \otimes_{R} M \rightarrow 0, \text { and } \\
0 \rightarrow & \operatorname{Hom}_{R}\left(B_{i}, F\right) \rightarrow \operatorname{Hom}_{R}\left(B_{i}, G_{i}\right) \rightarrow \operatorname{Hom}_{R}\left(B_{i}, B_{i}\right) \rightarrow \operatorname{Hom}_{R}\left(M_{i}, M_{i}\right) \rightarrow 0 .
\end{aligned}
$$

Proof. (cf. [33, Lem. 3.1 and 3.10]). We apply $\operatorname{Hom}_{R}\left(B_{i},-\right)$ to

$$
0 \longrightarrow F \longrightarrow G_{i} \longrightarrow B_{i} \longrightarrow 0
$$

and using $M_{i} \simeq \operatorname{Ext}_{R}^{1}\left(B_{i}, R\right)$ we deduce the exact sequence

$$
0 \rightarrow \operatorname{Hom}\left(B_{i}, F\right) \rightarrow \operatorname{Hom}_{R}\left(B_{i}, G_{i}\right) \rightarrow \operatorname{Hom}_{R}\left(B_{i}, B_{i}\right) \rightarrow F \otimes_{R} M_{i} \rightarrow G_{i} \otimes_{R} M_{i} .
$$


Hence we get the lemma by applying $\operatorname{Hom}\left(-, M_{i}\right)$ to $(2.8)$ and $(-) \otimes_{R} M$ to (2.11).

Proposition 2.4. Set $K_{i}:={ }_{0} \operatorname{hom}\left(B_{i-1}, R\left(a_{t+i-2}\right)\right)$ and $\ell_{i}:=\sum_{j=0}^{t+i-2} a_{j}-\sum_{q=1}^{t} b_{q}$. Letting $h_{i-3}:=2 a_{t+i-2}-\ell_{i}+n$ for $3 \leq i \leq c$ and $\left(\begin{array}{l}a \\ n\end{array}\right)=0$ if $a<n$ we have

$K_{i+3}=\sum_{\substack{r+s=i \\ r, s \geq 0}} \sum_{\substack{0 \leq i_{1}<\ldots<i_{r} \leq t+i \\ 1 \leq j_{1} \leq \ldots \leq j_{s} \leq t}}(-1)^{i-r}\left(\begin{array}{c}h_{i}+a_{i_{1}}+\cdots+a_{i_{r}}+b_{j_{1}}+\cdots+b_{j_{s}} \\ n\end{array}\right)$ for $0 \leq i \leq c-3$,

e.g. $K_{3}=\left(\begin{array}{c}h_{0} \\ n\end{array}\right)$ and $K_{4}=\sum_{j=0}^{t+1}\left(\begin{array}{c}h_{1}+a_{j} \\ n\end{array}\right)-\sum_{i=1}^{t}\left(\begin{array}{c}h_{1}+b_{i} \\ n\end{array}\right)$. Moreover if ${ }_{0} \operatorname{hom}(M, M)=1$ and the entries of the last $c-2$ columns belong to $\mathfrak{m}$ (e.g. $\mathcal{A}$ is minimal), then

$$
\operatorname{aut}\left(B_{c}\right):={ }_{0} \operatorname{hom}\left(B_{c}, B_{c}\right)=1+K_{3}+K_{4}+\cdots+K_{c} .
$$

Proof. Using (2.6) we get a resolution of $M_{i-1}$ which due to (2.8) leads to the following resolution:

$$
0 \rightarrow E_{i-3} \rightarrow \cdots \rightarrow E_{j} \rightarrow \cdots \rightarrow E_{0} \rightarrow B_{i-1}^{*} \rightarrow 0
$$

where $E_{j}:=\wedge^{t+j+1} G^{*} \otimes S_{j}(F) \otimes \wedge^{t} F$. Taking dimensions of the degree $a_{t+i-2}$ part of the free modules in this resolution and using $K_{i}=\operatorname{dim} B_{i-1}^{*}\left(a_{t+i-2}\right)_{0}$, we get that $K_{i}$ coincides with the sum of binomials appearing in the proposition (see [33, p.2882] for details).

Now dualizing the exact sequence $0 \rightarrow R\left(a_{t+c-2}\right) \rightarrow B_{c} \rightarrow B_{c-1} \rightarrow 0$, we get

$$
0 \rightarrow \operatorname{Hom}\left(B_{c-1}, R\right) \rightarrow \operatorname{Hom}_{R}\left(B_{c}, R\right) \rightarrow R\left(-a_{t+c-2}\right) \rightarrow M_{c-1} \rightarrow M_{c} \rightarrow 0
$$

by (2.8). Combining with (2.9) we get the exact sequence

$$
0 \rightarrow \operatorname{Hom}\left(B_{c-1}, R\right) \rightarrow \operatorname{Hom}_{R}\left(B_{c}, R\right) \rightarrow I_{D_{c-1}}\left(-a_{t+c-2}\right) \rightarrow 0
$$

which implies the vanishing of the lower down-arrows in the following commutative diagram

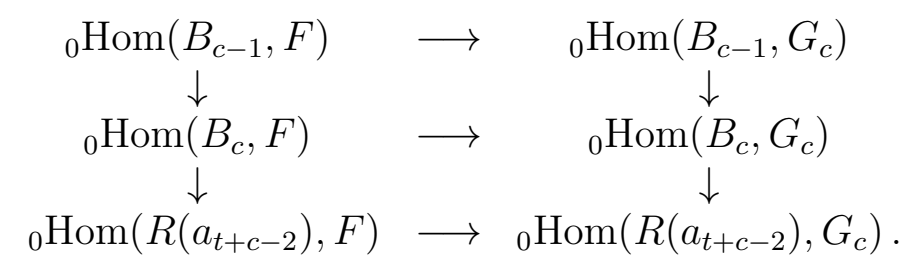

It follows that the upper down-arrows are bijections. Since $\operatorname{Hom}\left(B_{c-1},-\right)$ is exact on $0 \rightarrow R\left(a_{t+c-2}\right) \rightarrow G_{c} \rightarrow G_{c-1} \rightarrow 0$ (because $\left.\operatorname{Ext}^{1}\left(B_{c-1},-\right) \cong M_{c-1} \otimes(-)\right)$ we get

$$
{ }_{0} \operatorname{hom}\left(B_{c}, G_{c}\right)-{ }_{0} \operatorname{hom}\left(B_{c}, F\right)={ }_{0} \operatorname{hom}\left(B_{c-1}, G_{c-1}\right)-{ }_{0} \operatorname{hom}\left(B_{c-1}, F\right)+K_{c} .
$$

By Lemma 2.3 and the assumption ${ }_{0} \operatorname{hom}\left(M_{c}, M_{c}\right)=1$, we have

$$
\operatorname{aut}\left(B_{c}\right)=1+{ }_{0} \operatorname{hom}\left(B_{c}, G_{c}\right)-{ }_{0} \operatorname{hom}\left(B_{c}, F\right),
$$

and since there is a corresponding expression for aut $\left(B_{c-1}\right)$ we get

$$
\operatorname{aut}\left(B_{c}\right)=1+K_{c}+\operatorname{aut}\left(B_{c-1}\right)-{ }_{0} \operatorname{hom}\left(M_{c-1}, M_{c-1}\right)
$$

by (2.12). $D_{c-1}$ is, however, good determinantal because $I_{t}\left(\mathcal{A}_{c-1}\right) \subset I_{t}\left(\mathcal{A}_{c}\right) \subset I_{t-1}\left(\mathcal{A}_{c-1}\right)$ and $\operatorname{codim}_{D_{c-1}} D_{c}=1$. Indeed all $D_{j}, 2 \leq j \leq c-1$ are good determinantal for the same reason and we get ${ }_{0} \operatorname{hom}\left(M_{j}, M_{j}\right)=1$ for $2 \leq j \leq c-1$ by Remark 2.2. This simplifies (2.13) which holds also if we replace $c$ by $j+1$ in (2.13). Then we conclude the proof by induction using that $\operatorname{aut}\left(B_{2}\right)={ }_{0} \operatorname{hom}\left(I_{D_{2}}, I_{D_{2}}\right)=1$. 
Finally recall that if $J=I_{t-1}(\mathcal{A})$ and $\operatorname{dim} A>0$ then $X=\operatorname{Proj}(A)$ is a local complete intersection (l.c.i.) in $X-V(J)$. In the following we always take $Z \supset V(J)$ and $U=X-Z$, i.e. so that $X \hookrightarrow \mathbb{P}^{n}$ is an l.c.i. in $U$. Since the $1^{\text {st }}$ Fitting ideal of $M$ is equal to $J$, we get that $\tilde{M}$ and $\widetilde{I / I^{2}}$ are locally free on $X-V(J)$, cf. [50, Lem. 1.8] and [3, Lem. 1.4.8]. Note that $\operatorname{depth}_{J} A \geq \operatorname{codim}_{X} \operatorname{Sing}(X)$ which we use in the following.

Remark 2.5. Let $X=X_{c}, Y=X_{c-1}$ and let $\alpha$ be a positive integer. If $X$ is general in $W(\underline{b} ; \underline{a}) \neq \emptyset$ and $a_{i-\min (\alpha, t)}-b_{i} \geq 0$ for $\min (\alpha, t) \leq i \leq t$, then

$$
\operatorname{codim}_{X_{j}} \operatorname{Sing}\left(X_{j}\right) \geq \min \{2 \alpha-1, j+2\} \text { for } 2 \leq j \leq c,
$$

cf. the arguments in [33, Rem. 2.7] which use [7, 51]. In particular letting $\alpha=3$ we get that $X \hookrightarrow \mathbb{P}^{n}$ (resp. $Y \hookrightarrow \mathbb{P}^{n}$ and $X \hookrightarrow Y$ ) are l.c.i.s outside a subset $Z \subset X$ of codimension at least $\min (5, c+2)$ (resp. $\min (4, c))$ in $X$. Indeed we may take $Z=V\left(I_{t-1}\left(\mathcal{A}_{c-1}\right)\right)$ for the statement involving $X$ and $Y$ because (2.10) implies that $\mathcal{I}_{X / Y}$ is locally free on $Y-Z$, noting that $Z \subset V\left(I_{t}(\mathcal{A})\right)=X$. Moreover if $a_{i-2}-b_{i} \geq 0$ for $2 \leq i \leq t$, then $X \hookrightarrow \mathbb{P}^{n}$ (resp. $Y \hookrightarrow \mathbb{P}^{n}$ and $X \hookrightarrow Y$ ) are l.c.i.s outside a subset $Z \subset X$ of codimension at least $\min (3, c+2)$ (resp. $\min (2, c))$. Notice that we interpret $I(Z)$ as $\mathfrak{m}$ if $Z=\emptyset$.

2.3. The dimension of the determinantal locus. In [31] we proved that the dimension of a non-empty $W(\underline{b} ; \underline{a})$ in the case $a_{i-2}-b_{i} \geq 0$ for $i \geq 2$ and $n-c \geq 1$ is given by

$$
\operatorname{dim} W_{s}(\underline{b} ; \underline{a})=\operatorname{dim} W(\underline{b} ; \underline{a})=\lambda_{c}+K_{3}+K_{4}+\ldots+K_{c},
$$

where $K_{i}$ is defined in Proposition 2.4 and $\lambda_{c}$ is defined by

$$
\lambda_{c}:=\sum_{i, j}\left(\begin{array}{c}
a_{j}-b_{i}+n \\
n
\end{array}\right)+\sum_{i, j}\left(\begin{array}{c}
b_{i}-a_{j}+n \\
n
\end{array}\right)-\sum_{i, j}\left(\begin{array}{c}
a_{i}-a_{j}+n \\
n
\end{array}\right)-\sum_{i, j}\left(\begin{array}{c}
b_{i}-b_{j}+n \\
n
\end{array}\right)+1
$$

where the indices belonging to $a_{j}$ (resp. $b_{i}$ ) range over $0 \leq j \leq t+c-2($ resp. $1 \leq i \leq t)$.

Remark 2.6. Note that we assume (2.4) and delete columns from the right-hand side for (2.15) to hold. From the proof of Proposition 2.4 we see that we also need all algebras $D_{i}$ in $R \rightarrow D_{2} \rightarrow D_{3} \ldots \rightarrow D_{c}=A$ to be standard determinantal. If $D_{i}$ is standard determinantal and we delete a column whose entries belong to $\mathfrak{m}$, then $D_{i-1}$ is standard determinantal by [2], see Proposition 5.1 for deleting columns containing units. In particular (2.15) holds if the entries of the last $c-2$ columns of $\mathcal{A}$ belong to $\mathfrak{m}$ (e.g. if $\mathcal{A}$ is minimal).

Using, however, the Hilbert function, $H_{M}(-)$, of $M$ and assuming ${ }_{0} \operatorname{hom}(M, M)=1$, we have by [31, Rem. 3.9], for $\mathcal{A}$ possibly non-minimal, that

$$
\operatorname{dim} W_{s}(\underline{b} ; \underline{a})=\operatorname{dim} W(\underline{b} ; \underline{a})=\sum_{j=0}^{t+c-2} H_{M}\left(a_{j}\right)-\sum_{i=1}^{t} H_{M}\left(b_{i}\right)+1 .
$$

For zero-dimensional determinantal schemes $(n-c=0)$ we have the following.

Remark 2.7. (i) Assume $a_{0}>b_{t}$. Then (2.15) holds provided $3 \leq c \leq 5$ (resp. $c>5$ ) and $a_{t+c-2}>a_{t-2}$ (resp. $a_{t+3}>a_{t-2}$ ) by [34, Thm. 3.2], see Sec. 6 for a generalization.

(ii) If $\mathcal{A}$ is a linear $2 \times(c+1)$ matrix, we showed in [29, Ex. 3.3] that $\operatorname{dim} W(\underline{b} ; \underline{a})$ is strictly smaller that the right-hand side of (2.15) for every $c>2$. To our knowledge this is the only known case where the equality of (2.15) fails to hold when $n=c$. 
2.4. The determinantal locus as components of Hilbert schemes. It was shown in [31] that $\overline{W(\underline{b} ; \underline{a})}$, for $W(\underline{b} ; \underline{a}) \neq \emptyset$, is an irreducible component of $\operatorname{Hilb}^{p}\left(\mathbb{P}^{n}\right)$ provided $n-c \geq 2$ and $a_{i-\min (3, t)}-b_{i} \geq 0$ for $\min (3, t) \leq i \leq t$. If $n-c \leq 1$ then $\overline{W(\underline{b} ; \underline{a})}$ may fail to be an irreducible component. Indeed $\overline{W(0,0 ; 1,1, \ldots, 1,2)}$ is not an irreducible component of $\operatorname{GradAlg}(H)$ for all $c \geq 3$ both when $n=c$ and $n=c+1$, see [29, Ex. 4.1]. In both cases the $h$-vector of a general $A$ is $(1, c, c)$, e.g. the Hilbert function is given by $\left(\operatorname{dim} A_{i}\right)_{i=0}^{\infty}=(1, c+1,2 c+1,2 c+1, \ldots)$ if $n=c$.

The reason why $\overline{W(\underline{b} ; \underline{a})}$ is not a component is that there exist determinantal rings allowing "deformations that do not come from deforming the matrix $\mathcal{A}$ ". Let us recall this notion. We briefly say "T a local ring" (resp. " $T$ artinian") for a local $k$-algebra $\left(T, \mathfrak{m}_{T}\right)$ essentially of finite type over $k=T / \mathfrak{m}_{T}$ (resp. of finite type over $k=T / \mathfrak{m}_{T}$ such that $\mathfrak{m}_{T}^{r}=0$ for some $r \in \mathbb{Z}$ ). The local deformation functor $\operatorname{Def}_{A / R}$ is defined for each artinian $T$ as the set of graded ( $T$-flat) deformations $A_{T}$ of $A$ to $T$ (i.e. $A_{T} \otimes_{T} k=A$ ). Moreover " $T \rightarrow S$ is called a small artinian surjection" if there is a morphism $\left(T, \mathfrak{m}_{T}\right) \rightarrow\left(S, \mathfrak{m}_{S}\right)$ of local artinian $k$-algebras whose kernel $\mathfrak{a}$ satisfies $\mathfrak{a} \cdot \mathfrak{m}_{T}=0$.

If $T$ is a local ring, we let $\mathcal{A}_{T}=\left(f_{i j, T}\right)$ be a matrix of homogeneous polynomials of the graded algebra $R_{T}:=R \otimes_{k} T$, satisfying $f_{i j, T} \otimes_{T} k=f_{i j}$ and $\operatorname{deg} f_{i j, T}=a_{j}-b_{i}$ for all $i, j$. Here all elements of $T$ are considered to be of degree zero. Once having $\mathcal{A}_{T}$, it induces a morphism

$$
\varphi_{T}: F_{T}:=\oplus_{i=1}^{t} R_{T}\left(b_{i}\right) \rightarrow G_{T}:=\oplus_{j=0}^{t+c-2} R_{T}\left(a_{j}\right) .
$$

Lemma 2.8. If $A=R / I_{t}(\mathcal{A})$ is standard determinantal, then $A_{T}:=R_{T} / I_{t}\left(\mathcal{A}_{T}\right)$ and $M_{T}:=$ coker $\varphi_{T}^{*}$ are graded deformations of $A$ and $M$ respectively for every choice of $\mathcal{A}_{T}$, $T$ a local ring. Moreover every graded deformation of $M$ is of the form $M_{T}$ for some $\mathcal{A}_{T}$.

Proof. See [29, Lem. 4.2] for a proof. Indeed $A_{T}=R_{T} / I_{t}\left(\mathcal{A}_{T}\right)$ and $M_{T}$ are $T$-flat because all maps in their Eagon-Northcott and Buchsbaum-Rim complexes are defined in terms of $\mathcal{A}_{T}$, and these complexes become exact after tensoring by $k$ over $T$. Note also that the final statement in the lemma follows immediately from $M=\operatorname{coker} \varphi^{*}$.

The final statement may be formulated as "every graded deformation of $M$ to a local ring $T$ comes from deforming $\mathcal{A}$ ". This may not hold for $A$.

Definition 2.9. Let $A=R / I_{t}(\mathcal{A})$. We say "every deformation of $A$ (or $X$ if $\operatorname{dim} A>1$, see (2.1) ) comes from deforming $\mathcal{A}$ " if for every local ring $T$ and every graded deformation $R_{T} \rightarrow A_{T}$ of $R \rightarrow A$ to $T$, then $A_{T}$ is of the form $A_{T}=R_{T} / I_{t}\left(\mathcal{A}_{T}\right)$ for some $\mathcal{A}_{T}$ as above.

Lemma 2.10. Let $A=R / I_{t}(\mathcal{A})$ be a standard determinantal ring, $(A) \in W_{s}(\underline{b} ; \underline{a})$. If every deformation of $A$ comes from deforming $\mathcal{A}$, then $A$ is unobstructed (i.e. $\operatorname{Def}_{A / R}$ is smooth). Moreover $\overline{W_{s}(\underline{b} ; \underline{a})}$ is an irreducible component of $\operatorname{GradAlg}(H)$.

Proof. See [29, Lem. 4.4], only replacing $\operatorname{Hilb}\left(\mathbb{P}^{n}\right)$ by $\operatorname{GradAlg}(H)$ in its proof.

Remark 2.11. By these lemmas we get $T$-flat determinantal schemes by just parameterizing the polynomials of $\mathcal{A}$ over a local ring $T$, see Rem. 4.5 of [29] and Laksov's papers [38, 37] for somewhat similar results for more general determinantal schemes. 


\section{DEFORMATIONS OF MODULES AND DETERMINANTAL RINGS}

The main goal of this section is to generalize to $A$ artinian the close relationship between the local deformation functor, $\operatorname{Def}_{M / R}$, of the graded $R$-module $M=\operatorname{coker} \varphi^{*}$ and the corresponding local functor, $\operatorname{Def}_{A / R}$, of graded deformations of the standard determinantal $\operatorname{ring} A=R / I_{t}(\mathcal{A})$. Note that $I:=I_{t}(\mathcal{A})=\operatorname{ann}(M)$ by [5]. In [31] these functors were shown to be isomorphic (resp. the first a natural subfunctor of the latter) provided $\operatorname{dim} X \geq 2$ (resp. $\operatorname{dim} X=1)$ and $X=\operatorname{Proj}(A)$ general and good determinantal. The comparison between these deformation functors relied on understanding the spectral sequence $E_{2}^{p, q}:=\operatorname{Ext}_{A}^{p}\left(\operatorname{Tor}_{q}^{R}(A, M), M\right) \Rightarrow \operatorname{Ext}_{R}^{p+q}(M, M)$ and its induced 5-term exact sequence

$$
0 \rightarrow \operatorname{Ext}_{A}^{1}(M, M) \rightarrow \operatorname{Ext}_{R}^{1}(M, M) \stackrel{\delta}{\longrightarrow} E_{2}^{0,1} \rightarrow \operatorname{Ext}_{A}^{2}(M, M) \rightarrow \operatorname{Ext}_{R}^{2}(M, M) \rightarrow .
$$

Indeed $\operatorname{Tor}_{1}^{R}(A, M) \simeq I \otimes_{R} M$ implies $E_{2}^{0,1} \simeq \operatorname{Hom}_{R}\left(I, \operatorname{Hom}_{R}(M, M)\right)$ in general, and since $\operatorname{Hom}_{A}(M, M) \simeq A$ provided $A$ is good determinantal (i.e. $\operatorname{depth}_{I_{t-1}(\mathcal{A}) A} A \geq 1$ ) by [33, Lem. 3.2], it follows in this case that the morphism $\delta$ of (3.1) induces a natural map

$$
{ }_{0} \operatorname{Ext}_{R}^{1}(M, M) \longrightarrow\left(E_{2}^{0,1}\right)_{0} \simeq{ }_{0} \operatorname{Hom}_{R}(I, A)
$$

between the tangent spaces of $\operatorname{Def}_{M / R}$ and $\operatorname{Def}_{A / R}$. For $A$ standard determinantal, there is still a natural map $e_{M}(T): \operatorname{Def}_{M / R}(T) \rightarrow \operatorname{Def}_{A / R}(T), T$ artinian, obtained by taking a matrix $\mathcal{A}_{T}$ whose corresponding morphism has $M_{T}$ as cokernel and letting $A_{T}:=R_{T} / I_{t}\left(\mathcal{A}_{T}\right)$ (see Lemma 2.8). Since matrices inducing the same $M_{T}$ define the same ideal of maximal minors (Fitting's lemma, [10, Cor. 20.4]), this morphism is well-defined.

Remark 3.1. The main difficulty in generalizing the comparison above to an artinian $A$ is that $A \rightarrow \operatorname{Hom}_{A}(M, M)$ is no longer an isomorphism (for $A$ not a c.i.) which implies that the tangent map of $\operatorname{Def}_{M / R} \rightarrow \operatorname{Def}_{A / R}$ may not be the map in (3.2), i.e. $\left(E_{2}^{0,1}\right)_{0} \simeq$ ${ }_{0} \operatorname{Hom}_{R}(I, A)$ may fail. The morphism $\operatorname{Def}_{M / R} \rightarrow \operatorname{Def}_{A / R}$ is, however, well-defined also in the artinian case, and since $I_{t}(\mathcal{A})=\operatorname{ann}(M)$ implies that $A \rightarrow \operatorname{Hom}_{A}(M, M)$ is injective and the functors are pro-representable provided ${ }_{0} \operatorname{Hom}_{A}(M, M) \simeq k$, we shall see that we are able to generalize the comparison.

Definition 3.2. Let $A=R / I_{t}(\mathcal{A})$ be a standard determinantal ring and let $\underline{\ell}$ be the category of artinian $k$-algebras (cf. the text before (2.18) ). Then the local deformation functor $\operatorname{Def}_{A \in W_{s}(\underline{b} ; \underline{a})}$, defined on $\underline{\ell}$, is the subfunctor of $\operatorname{Def}_{A / R}$ given by:

$\operatorname{Def}_{A \in W_{s}(\underline{b} ; \underline{a})}(T)=\left\{A_{T} \in \operatorname{Def}_{A / R}(T) \mid A_{T}=R_{T} / I_{t}\left(\mathcal{A}_{T}\right)\right.$ for some matrix $\mathcal{A}_{\mathrm{T}} \operatorname{lifting} \mathcal{A}$ to $\left.T\right\}$.

With this definition it is obvious that the natural map $e_{M}: \operatorname{Def}_{M / R} \rightarrow \operatorname{Def}_{A / R}$ defined above for any $T \in \mathrm{ob}(\underline{\ell})$ factors via $\operatorname{Def}_{A \in W_{s}(\underline{b} ; \underline{a})} \hookrightarrow \operatorname{Def}_{A / R}$. Moreover we have

Lemma 3.3. If $D:=k[\epsilon] /\left(\epsilon^{2}\right)$ then the degree zero part, $\delta_{0}(M)$, of the connecting homomorphism $\delta$ of (3.1) factors through $e_{M}(D): \operatorname{Def}_{M / R}(D) \rightarrow \operatorname{Def}_{A / R}(D)$, i.e. $\delta_{0}(M)$ is the composition

$$
{ }_{0} \operatorname{Ext}_{R}^{1}(M, M) \stackrel{e_{M}(D)}{\longrightarrow}{ }_{0} \operatorname{Hom}_{R}(I, A) \stackrel{i_{A, M}}{\longrightarrow}{ }_{0} \operatorname{Hom}_{R}\left(I, \operatorname{Hom}_{A}(M, M)\right) \simeq\left(E_{2}^{0,1}\right)_{0}
$$

where the map in the middle is induced by $A \hookrightarrow \operatorname{Hom}_{A}(M, M), 1 \in A \mapsto i d_{M}$. 
Proof. We know that $e_{M}(D):{ }_{0} \operatorname{Ext}_{R}^{1}(M, M) \rightarrow{ }_{0} \operatorname{Hom}_{R}(I, A)$ is well-defined. To describe it, take any $\bar{\eta} \in{ }_{0} \operatorname{Ext}_{R}^{1}(M, M)$ and let $\eta^{\prime} \in{ }_{0} \operatorname{Hom}\left(G^{*}, M\right)$ represent $\bar{\eta}$ and $\eta \in{ }_{0} \operatorname{Hom}\left(G^{*}, F^{*}\right)$ map to $\eta^{\prime}$, cf. (3.3). A maximal minor $f_{1}$ corresponds to choosing $t$ columns of $\mathcal{A}$ which we for simplicity suppose is obtained by deleting the last $c-1$ columns of $\mathcal{A}$. With notations as in Sec. $2, f_{1}$ is the determinant of $\varphi_{1}^{*}: G_{1}^{*}=\oplus_{j=0}^{t-1} R\left(-a_{j}\right) \rightarrow F^{*}=$ $\oplus_{i=1}^{t} R\left(-b_{i}\right)$. By definition $e_{M}(D)(\bar{\eta})$ is given by the ideal generated by maximal minors of $\varphi^{*}+\epsilon \eta$. Let $I_{1}$ be the principal ideal generated by $f_{1}$. Since it is easy to compute $\operatorname{det}\left(\varphi_{1}^{*}+\epsilon \eta_{1}\right)$ where $\eta_{1}$ is the composition, $G_{1}^{*} \hookrightarrow G^{*} \stackrel{\eta}{\longrightarrow} F^{*}$, of the natural inclusion $G_{1}^{*} \hookrightarrow G^{*}$ by $\eta$, we get that the image of $e_{M}(D)(\bar{\eta})$ in ${ }_{0} \operatorname{Hom}_{R}\left(I_{1}, A\right) \simeq A_{\left(\operatorname{deg} f_{1}\right)}$ via the natural map $I_{1} \hookrightarrow I$ is $\operatorname{tr}\left(\varphi_{1}^{a} \cdot \eta_{1}\right) \otimes_{R} 1$ where $1 \in A$ is the unity, $\varphi_{1}^{a}$ is the adjoint of $\varphi_{1}^{*}$ and $\operatorname{tr}$ the trace map.

Let $M_{1}=\operatorname{coker} \varphi_{1}^{*}$ and let $\pi: M_{1} \rightarrow M$ be the canonical surjection. To see that $\delta_{0}(M)=i_{A, M} \cdot e_{M}(D)$ it suffices to check that the image of $\delta_{0}(M)(\bar{\eta})$ via the composition

$$
{ }_{0} \operatorname{Hom}_{R}(I, \operatorname{Hom}(M, M)) \rightarrow{ }_{0} \operatorname{Hom}_{R}\left(I_{1}, \operatorname{Hom}(M, M)\right) \hookrightarrow{ }_{0} \operatorname{Hom}_{R}\left(I_{1}, \operatorname{Hom}\left(M_{1}, M\right)\right)
$$

is $\operatorname{tr}\left(\varphi_{1}^{a} \cdot \eta_{1}\right) \otimes_{R} \pi$ because the maximal minors provide a surjection $\oplus R\left(-n_{i}\right) \rightarrow I$ and hence an injection ${ }_{0} \operatorname{Hom}_{R}(I, \operatorname{Hom}(M, M)) \rightarrow{ }_{0} \operatorname{Hom}_{R}\left(\oplus R\left(-n_{i}\right), \operatorname{Hom}(M, M)\right)$ (and think of $f_{1}$ as an arbitrary minor). Then we can conclude the proof by the functoriality of connecting homomorphisms and Ile's result for $\delta_{0}\left(M_{1}\right)$ in [24]. Indeed we have a commutative diagram

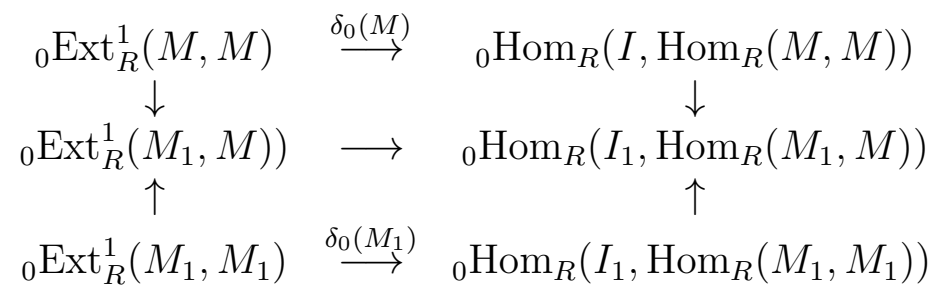

of three connecting homomorphisms. If $A_{1}=R / I_{1}$ then $\delta_{0}\left(M_{1}\right)\left(\overline{\eta_{1}}\right)=\operatorname{tr}\left(\varphi_{1}^{a} \cdot \eta_{1}\right) \otimes_{R} i d_{M_{1}}$ by [24, Prop. 2], whence maps to $\operatorname{tr}\left(\varphi_{1}^{a} \cdot \eta_{1}\right) \otimes_{R} \pi$ via the right up-arrow. Here $\overline{\eta_{1}} \in$ ${ }_{0} \operatorname{Ext}_{R}^{1}\left(M_{1}, M_{1}\right)$ is represented by the composition $G_{1}^{*} \stackrel{\eta_{1}}{\longrightarrow} F^{*} \rightarrow M_{1}$. Since the elements $\bar{\eta} \in{ }_{0} \operatorname{Ext}_{R}^{1}(M, M)$ and $\overline{\eta_{1}} \in{ }_{0} \operatorname{Ext}_{R}^{1}\left(M_{1}, M_{1}\right)$ map to the same element in ${ }_{0} \operatorname{Ext}_{R}^{1}\left(M_{1}, M\right)$ (the one represented by the composition $G_{1}^{*} \stackrel{\eta_{1}}{\longrightarrow} F^{*} \rightarrow M$ ) the proof is complete.

First we will find the dimension of the pro-representing object of $\operatorname{Def}_{M / R}$, i.e. we need to generalize [31, Thm. 3.2] by weakening its conditions so that it applies to an artinian $A$. Indeed we have (and see Proposition 2.4 and Remark 2.6 for computing the dimension).

Theorem 3.4. Let $A=R / I_{t}(\mathcal{A})$ be standard determinantal and let $M=$ coker $\varphi^{*}$. Then $M$ is unobstructed, i.e. $\operatorname{Def}_{M / R}$ is formally smooth. Moreover if ${ }_{0} \operatorname{Hom}_{A}(M, M) \simeq k$, then Def $_{M / R}$ is pro-representable, and the pro-representing object $H(M / R)$ of $\operatorname{Def}_{M / R}$ satisfies

$$
\operatorname{dim} H(M / R)=\operatorname{dim}_{0} \operatorname{Ext}_{R}^{1}(M, M)=\lambda_{c}+K_{3}+K_{4}+\ldots+K_{c} .
$$

Moreover we have $\operatorname{dim}{ }_{0} \operatorname{Ext}_{R}^{1}(M, M)=\sum_{j=0}^{t+c-2} H_{M}\left(a_{j}\right)-\sum_{i=1}^{t} H_{M}\left(b_{i}\right)+1$.

Proof. For the unobstructedness of $M$, see [31, Thm. 3.1] or Ile's PhD thesis [22, ch. 6].

To see the dimension formula we claim that there is an exact sequence

$$
0 \rightarrow{ }_{0} \operatorname{Hom}_{R}(M, M) \rightarrow{ }_{0} \operatorname{Hom}_{R}\left(F^{*}, M\right) \rightarrow{ }_{0} \operatorname{Hom}_{R}\left(G^{*}, M\right) \rightarrow{ }_{0} \operatorname{Ext}_{R}^{1}(M, M) \rightarrow 0 .
$$


Indeed the map $d_{1}: \wedge^{t+1} G^{*} \otimes S_{0}(F) \otimes \wedge^{t} F \rightarrow G^{*}$ appearing in the Buchsbaum-Rim complex (2.6), takes an element of $\wedge^{t+1} G^{*} \otimes S_{0}(F) \otimes \wedge^{t} F$ to a linear combination of maximal minors with coefficients in $G^{*}$ because $I_{t}(\mathcal{A})=\operatorname{im}\left(\wedge^{t} G^{*} \otimes S_{0}(F) \otimes \wedge^{t} F \rightarrow R\right)$ is generated by maximal minors, whence ${ }_{0} \operatorname{Hom}_{R}\left(d_{1}, M\right)=0$ because $I=\operatorname{ann}(M)$. So if we apply ${ }_{0} \operatorname{Hom}_{R}(-, M)$ to (2.6) , we get (3.3) by the definition of ${ }_{0} \operatorname{Ext}_{R}^{i}(M, M)$.

Taking dimensions of the groups in (3.3) and using (2.7) and ${ }_{0} \operatorname{hom}(M, M)=1$ we get the latter dimension formula for $\operatorname{dim}{ }_{0} \operatorname{Ext}_{R}^{1}(M, M)$. Moreover if we apply the exact functors ${ }_{0} \operatorname{Hom}_{R}\left(F^{*},-\right)$ and ${ }_{0} \operatorname{Hom}_{R}\left(G^{*},-\right)$ onto (2.8) with $i=c$ we get

$$
{ }_{0} \operatorname{hom}_{R}\left(G^{*}, M\right)-{ }_{0} \operatorname{hom}_{R}\left(F^{*}, M\right)=\lambda_{c}-1+{ }_{0} \operatorname{hom}_{R}\left(G^{*}, B^{*}\right)-{ }_{0} \operatorname{hom}_{R}\left(F^{*}, B^{*}\right)
$$

by using the definition (2.16) of $\lambda_{c}$. Hence we get the dimension formula (i.e. the rightmost equality) of Theorem 3.4 provided we can prove

$$
{ }_{0} \operatorname{hom}_{R}(B, G)-{ }_{0} \operatorname{hom}_{R}(B, F)=K_{3}+\ldots+K_{c} .
$$

This follows from Proposition 2.4 and the second exact sequence of Lemma 2.3 .

Finally the condition ${ }_{0} \operatorname{Hom}_{A}(M, M) \simeq k$ allows us to lift automorphisms, i.e. $\operatorname{Def}_{M / R}$ is pro-representable, cf. [18, Thm. 19.2], whence $\operatorname{dim} H(M / R)=\operatorname{dim}{ }_{0} \operatorname{Ext}_{R}^{1}(M, M)$ by the smoothness of $\operatorname{Def}_{M / R}$.

Let $D:=k[\epsilon] /\left(\epsilon^{2}\right)$ be the dual numbers and denote the dimension in Theorem 3.4 by

$$
\lambda:=\operatorname{dim}{ }_{0} \operatorname{Ext}_{R}^{1}(M, M)=\lambda_{c}+K_{3}+K_{4}+\ldots+K_{c} .
$$

Recalling that $W_{s}(\underline{b} ; \underline{a})$ is a certain quotient of an open irreducible set in the affine scheme $\mathbb{V}=\operatorname{Hom}_{R}\left(G^{*}, F^{*}\right)$ parameterizing determinantal $k$-algebras (Proposition 2.1), we get

Theorem 3.5. Let $A=R / I, I=I_{t}(\mathcal{A})$, be a standard determinantal $k$-algebra and let $M=\operatorname{coker} \varphi^{*}$.

(i) If ${ }_{0} \operatorname{Hom}_{A}(M, M) \simeq k$ and ${ }_{0} \operatorname{Ext}_{A}^{1}(M, M)=0$ then

$$
\operatorname{Def}_{A \in W_{s}(\underline{b} ; \underline{a})} \simeq \operatorname{Def}_{M / R} \text {. }
$$

Hence $\operatorname{Def}_{A \in W_{s}(\underline{b} ; \underline{a})}$ is a formally smooth pro-representable functor and the pro-representing object has dimension

$$
\operatorname{dim} W_{s}(\underline{b} ; \underline{a})=\lambda_{c}+K_{3}+K_{4}+\ldots+K_{c},
$$

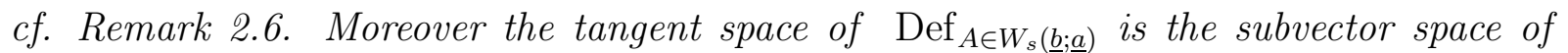
${ }_{0} \mathrm{Hom}_{R}(I, A)$ that corresponds to graded deformation $R_{D} \rightarrow A_{D}$ of $R \rightarrow A$ to $D$ of the form $A_{D}=R_{D} / I_{t}\left(\mathcal{A}_{D}\right)$ for some matrix $\mathcal{A}_{D}$ which lifts $\mathcal{A}$ to $D$.

(ii) If in addition ${ }_{0} \operatorname{Ext}_{A}^{2}(M, M)=0$, then $\operatorname{Def}_{M / R} \simeq \operatorname{Def}_{A \in W_{s}(\underline{b} ; \underline{a})} \simeq \operatorname{Def}_{A / R}$ and $\operatorname{Def}_{A / R}$ is formally smooth. Moreover every deformation of $A$ comes from deforming $\mathcal{A}$ (cf. Definition 2.9).

Proof. We already know that $\operatorname{Def}_{M / R}(T) \rightarrow \operatorname{Def}_{A \in W_{s}(\underline{b} ; \underline{a})}(T)$ is well-defined (Fitting's lemma) and obviously surjective for any $\left(T, \mathfrak{m}_{T}\right)$ in $\underline{\ell}$. To see the injectivity, let $M_{i}$ for $i=1,2$ represent two elements of $\operatorname{Def}_{M / R}(T)$ mapping to the same element $A_{T} \in$ $\operatorname{Def}_{A \in W_{s}(\underline{b} ; \underline{a})}(T)$. Since $M_{i}, i=1,2$ are cokernels of two morphisms given by matrices $\left(\mathcal{A}_{T}\right)_{1}$ and $\left(\mathcal{A}_{T}\right)_{2}$ lifting $\mathcal{A}$ to $T$, we have $A_{T}=R_{T} / I_{t}\left(\left(\mathcal{A}_{T}\right)_{1}\right)=R_{T} / I_{t}\left(\left(\mathcal{A}_{T}\right)_{2}\right)$, cf. Definition 3.2. To show the injectivity, we may by induction on $r \geq 1$ suppose 
$\mathfrak{m}_{T}^{r+1}=0$ and $\operatorname{Def}_{M / R}\left(T / \mathfrak{m}_{T}^{r}\right) \rightarrow \operatorname{Def}_{A \in W(\underline{b} ; \underline{a})}\left(T / \mathfrak{m}_{T}^{r}\right)$ injective. Noticing that the condition ${ }_{0} \operatorname{Hom}_{A}(M, M) \simeq k$ allows us to lift automorphisms of $M_{i} \otimes_{T} T / \mathfrak{m}_{T}^{r}$ to automorphisms of $M_{i}$, it follows from ${ }_{0} \operatorname{Ext}_{A}^{1}(M, M)=0$ that the graded deformations $M_{1}$ and $M_{2}$ represent the same element of $\operatorname{Def}_{M / R}(T)$, and the injectivity is proved.

Moreover using $\operatorname{Def}_{A \in W_{s}(\underline{b} ; \underline{a})} \simeq \operatorname{Def}_{M / R}$ and Theorem 3.4 we get that $\operatorname{Def}_{A \in W_{s}(\underline{b} ; \underline{a})}$ is prorepresentable and formally smooth, whence its pro-representing object $H$ satisfies $H \simeq$ $H(M / R)$ and since $H$ and its "universal family" $A_{H}$ are algebraizable by the proof of [31, Thm. 5.2] (or use the $2^{\text {nd }}$ paragraph of the proof of Theorem 4.8 of this paper to see that we get a morphism $\mathcal{O}_{\operatorname{GradAlg}(H),(A)} \rightarrow S, H \simeq \hat{S}$ with "universal family" $A_{S}:=R_{S} / I_{t}\left(\mathcal{A}_{S}\right)$, which extends to open subsets $V \subset U$, of $W_{s}(\underline{b} ; \underline{a})$ and $\operatorname{GradAlg}(H)$ respectively with $(A) \in V$ and $\left.\mathcal{O}_{V,(A)}=S\right)$, we get $\operatorname{dim} H=\operatorname{dim} W_{s}(\underline{b} ; \underline{a})$ by Theorem 3.4. The description of its tangent space follows from (3.1) and Definition 3.2 since ${ }_{0} \mathrm{Hom}_{R}(I, A)$ is the tangent space of $\operatorname{Def}_{A / R}$.

If $_{0} \operatorname{Ext}_{A}^{2}(M, M)=0$ then ${ }_{0} \operatorname{Ext}_{R}^{1}(M, M) \simeq\left(E_{2}^{0,1}\right)_{0} \simeq{ }_{0} \operatorname{Hom}_{R}\left(I, \operatorname{Hom}_{A}(M, M)\right)$ by (3.1). Hence the first part of the proof and $A \hookrightarrow \operatorname{Hom}_{A}(M, M)$ imply that all the injections in

$$
\operatorname{Def}_{M / R}(D) \hookrightarrow \operatorname{Def}_{A / R}(D) \simeq{ }_{0} \operatorname{Hom}_{R}(I, A) \hookrightarrow{ }_{0} \operatorname{Hom}_{R}\left(I, \operatorname{Hom}_{A}(M, M)\right)
$$

are isomorphisms of finite dimensional vector spaces. It follows that $\operatorname{Def}_{M / R} \rightarrow \operatorname{Def}_{A / R}$ is an isomorphism since it is bijective on tangent spaces and $\operatorname{Def}_{M / R}$ is formally smooth. Then we conclude the proof by arguing as in the proof of [31, Thm. 5.2].

Remark 3.6. The theorems of this section admit substantial generalizations with respect to $R$ being a polynomial ring. Indeed we may let $R$ be any graded quotient of a polynomial ring. The main reason for this is that the spectral sequence of this section, cf. (3.1), Fitting's lemma and the exactness of the Buchsbaum-Rim complex are valid with almost no assumption on $R$ (but we need to replace the binomials defining $\lambda_{c}$ and $K_{i}$ with their Hilbert functions; the final formula of Theorems 3.4 is, however, valid as stated).

\section{THE LOCUS OF DETERMINANTAL K-ALGEBRAS}

In this section we generalize [31, Theorems 5.5 and 5.8] concerning dimension and smoothness of $\operatorname{Grad} \operatorname{Alg}(H)$ along $W_{s}(\underline{b} ; \underline{a})$, to cover the artinian case. Indeed using that Theorem 3.5 extends [31, Thm. 5.2] by only assuming ${ }_{0} \operatorname{Hom}_{A}(M, M) \simeq k$ and $A$ standard determinantal instead of good determinantal, the generalizations in Theorems 4.1 and 4.6 from good to standard determinantal are rather immediate. In this section we also prove a new result (Theorem 4.8) to cover cases where $\operatorname{dim} W_{s}(\underline{b} ; \underline{a}) \neq \lambda$.

In the first theorem we let, as in [31],

$$
\operatorname{ext}^{2}(M, M):=\operatorname{dim} \operatorname{ker}\left({ }_{0} \operatorname{Ext}_{A}^{2}(M, M) \rightarrow{ }_{0} \operatorname{Ext}_{R}^{2}(M, M)\right), \quad \text { cf. (3.1) . }
$$

Clearly $\operatorname{ext}^{2}(M, M) \leq{ }_{0} \operatorname{ext}_{A}^{2}(M, M)$, and note that we below may replace $\operatorname{GradAlg}(H)$ with $\operatorname{Hilb}^{p}\left(\mathbb{P}^{n}\right)$ if $n-c \geq 1$, cf. the text accompanying (2.1) for explanations and notations.

Theorem 4.1. Let $A=R / I, I=I_{t}(\mathcal{A})$ be a standard determinantal $k$-algebra, i.e. $(A) \in W_{s}(\underline{b} ; \underline{a})$, let $M=\operatorname{coker} \varphi^{*}$ and suppose ${ }_{0} \operatorname{Hom}_{A}(M, M) \simeq k$ and ${ }_{0} \operatorname{Ext}_{A}^{1}(M, M)=0$. Then

$$
\operatorname{dim} W_{s}(\underline{b} ; \underline{a})=\lambda:=\lambda_{c}+K_{3}+K_{4}+\ldots+K_{c}
$$


cf. Remark 2.6. Moreover, for the codimension of $W_{s}(\underline{b} ; \underline{a})$ in $\operatorname{GradAlg}(H)$ in a neighborhood of $(A)$ we have

$$
\operatorname{dim}_{(A)} \operatorname{GradAlg}(H)-\operatorname{dim} W_{s}(\underline{b} ; \underline{a}) \leq \operatorname{dim}{ }_{0} \operatorname{Hom}(I, A)-\lambda \leq \operatorname{ext}^{2}(M, M),
$$

where the first inequality is an equality if and only if $\operatorname{Grad} \operatorname{Alg}(H)$ is smooth at $(A)$. In particular these conclusions hold if $\operatorname{dim} A \geq 3+\operatorname{dim} R / I_{t-1}(\mathcal{A})$, or if $\operatorname{dim} A \geq 2$ and $\operatorname{dim} R / I_{t-1}(\mathcal{B})=0$ where $\mathcal{B}$ is obtained from $\mathcal{A}$ by deleting some column of $\mathcal{A}$ (e.g. if $\operatorname{dim} A \geq 2, a_{i-2} \geq b_{i}$ for $2 \leq i \leq t$ and $A$ is general).

Proof. The $1^{\text {st }}$ conclusion follows from Theorem 3.5(i). For the statements on the codimension one knows that $\operatorname{dim}_{(A)} \operatorname{GradAlg}(H) \leq \operatorname{dim}{ }_{0} \operatorname{Hom}_{R}(I, A)$ with equality if and only if $A$ is unobstructed ([25, Thm. 1.5]). Using (3.1), (3.4) and the injection $A \hookrightarrow{ }_{0} \operatorname{Hom}_{A}(M, M)$, it follows that ${ }_{0} \operatorname{Hom}_{R}(I, A)-\lambda \leq \operatorname{dim}\left(E_{2}^{0,1}\right)_{0}-\lambda=\operatorname{ext}^{2}(M, M)$, and we get the next conclusion of Theorem 4.1. Now since $\operatorname{depth}_{I_{t-1}(\mathcal{A}) A} A=\operatorname{dim} A-\operatorname{dim} R / I_{t-1}(\mathcal{A})$ and $A$ good determinantal is equivalent to $\operatorname{depth}_{I_{t-1}(\mathcal{A}) A} A \geq 1$, the final sentence follows directly from [31, Theorem 5.5], and the parenthesis from Remark 2.5.

There are many artinian determinantal rings satisfying the conditions ${ }_{0} \operatorname{Hom}_{A}(M, M) \simeq$ $k$ and ${ }_{0} \operatorname{Ext}_{A}^{1}(M, M)=0$ of Theorem 4.1. After having computed many examples using Macaulay 2 the general picture when $a_{0}>b_{t}$ seems to be that the only artinian determinantal rings $A=R / I_{t}(\mathcal{A})$ that do not satisfy these conditions are those with matrix $\mathcal{A}$ that is linear except in one column $v \in R_{m}^{\oplus t}$ where the degree is $m \geq 2$, so $\mathcal{A}$ is of the form $[\mathcal{B}, v]$ where $\mathcal{B}$ is linear. The following example avoids this case, but $\mathcal{B}$ is otherwise quite close to being linear (the case where $\mathcal{B}$ is linear will be considered in Example 4.11). Since the codimension- 2 case is straightforward in this context, the first non-trivial case is $c=3$. We include a codimension- 4 example to see that it can be treated similarly.

Example 4.2. (determinantal artinian quotients of $R$, using Theorem 4.1)

(i) Let $R=k\left[x_{0}, x_{1}, x_{2}\right]$ and let $\mathcal{A}=[\mathcal{B}, v]$ be a general $2 \times 4$ matrix with linear (resp. quadratic) entries in the first and second (resp. third) column and where the entries of $v$ are polynomials of the same degree $m$, i.e. the degree matrix of $\mathcal{A}$ is $\left(\begin{array}{llll}1 & 1 & 2 & m \\ 1 & 1 & 2 & m\end{array}\right)$. The vanishing of all $2 \times 2$ minors defines an artinian ring with $h$-vector $(1,3,5,5, . ., 5,5,3)$, where the number of $5 s$ is $m-1$. For $m \geq 2$ one verifies that the first conditions of Theorem 4.1, i.e. that all conditions of Theorem 3.5 (i) hold, and it follows that $\overline{W_{s}(\underline{b} ; \underline{a})}$ is an irreducible subset of $\operatorname{GradAlg}(H)$ of dimension $\lambda_{3}+K_{3}$ which is 14 for $m \geq 3$ (or one may use (2.17) to find the dimension). For $m \geq 5$ one shows that ${ }_{0} \operatorname{hom}_{A}\left(I / I^{2}, A\right)=16$, and we get that the codimension of $\overline{W_{s}(\underline{b} ; \underline{a})}$ in $\operatorname{GradAlg}(H)$ is at most 2 by Theorem 4.1 , The dimensions of the ${ }_{0}$ Ext $^{i}$-groups above are computed by using Macaulay 2 (over the finite fields $\mathbb{Z}_{101}, \mathbb{Z}_{701}$ and $\mathbb{Z}_{3001}$ ), and strictly speaking only for $m \leq 10$, from which we clearly see the general pattern also for $m>10$. Note that the dimensions we found of the ${ }_{0} \mathrm{Ext}^{i}$-groups are independent of the characteristic of the fields and since field extensions are flat, they remain unchanged over any field containing one of these three fields. So the conclusions above hold at least when $k$ is the algebraic closure of $\mathbb{Z}_{p}, p \in\{101,701,3001\}$.

(ii) Let $R=k\left[x_{0}, x_{1}, x_{2}, x_{3}\right]$ and let $\mathcal{A}=[\mathcal{B}, v]$ be a general $2 \times 5$ matrix with degree matrix $\left(\begin{array}{lllll}1 & 1 & 1 & 2 & m \\ 1 & 1 & 1 & 2 & m\end{array}\right)$. The vanishing of all $2 \times 2$ minors defines an artinian ring with $h$ vector $(1,4,7,7, . ., 7,4)$, where the number of $7 s$ is $m-1$. For $m \geq 2$ one verifies that 
all conditions of Theorem 3.5(i) hold, and we get that $\overline{W_{s}(\underline{b} ; \underline{a})}$ is an irreducible subset of $\operatorname{Grad} \operatorname{Alg}(H)$ of dimension $\lambda_{4}+K_{3}+K_{4}$, which is equal to 25 if $m \geq 3$ (or one may use (2.17) to find the dimension). For $m \geq 5,{ }_{0} \operatorname{hom}_{A}\left(I / I^{2}, A\right)=33$, whence the codimension of $\overline{W_{s}(\underline{b} ; \underline{a})}$ in $\operatorname{Grad} \operatorname{Alg}(H)$ is at most 8 by Theorem 4.1. The computations of the ${ }_{0}$ Ext $^{i}$ groups are verified by Macaulay 2 for $m \leq 10$ over the fields $\mathbb{Z}_{101}, \mathbb{Z}_{701}$ and $\mathbb{Z}_{3001}$, but their dimensions are the same for any field containing one of these fields and for $m>10$.

Remark 4.3. In all examples of this section we have verified the dimensions of those ${ }_{0} \operatorname{Ext}_{A}^{i}(M, M)$ and ${ }_{0} \operatorname{Ext}_{A}^{i}\left(I / I^{2}, A\right), i \geq 0$ we need to apply Theorems 4.1, 4.6 and 4.8 by using Macaulay 2 over each of the ground fields $\mathbb{Z}_{p}$ corresponding to $p=101,701$ and $p=3001$. But we have also computed these dimensions for many other values of $p>10$, and it seems that they are independent of the characteristic of the field.

Sometimes Theorem 4.1 allows us to find the exact codimension of $\overline{W_{s}(\underline{b} ; \underline{a})}$ in $\operatorname{GradAlg}(H)$.

Example 4.4. (determinantal artinian quotients of $R=k\left[x_{0}, x_{1}, x_{2}\right]$, using Theorem 4.1)

(i) Let $\mathcal{A}$ be a general $2 \times 4$ matrix with quadratic (resp. linear) entries in the first (resp. second) row, i.e. with degree matrix $\left(\begin{array}{llll}2 & 2 & 2 & 2 \\ 1 & 1 & 1 & 1\end{array}\right)$. The vanishing of all $2 \times 2$ minors defines an artinian ring with $h$-vector $(1,3,6,4,1)$. Macaulay 2 computations (cf. Remark 4.3) show that all conditions of Theorem [3.5(i) hold, and it follows that $\overline{W_{s}(\underline{b} ; \underline{a})}$ is an irreducible subset of $\operatorname{Grad} \operatorname{Ag}(H)$ of dimension $\lambda_{3}+K_{3}=16$. Moreover we get that $A$ is unobstructed by (2.2). Indeed both ${ }_{0} \operatorname{Ext}_{A}^{1}\left(I / I^{2}, A\right)=0$ (verified by Macaulay 2) and ${ }_{0} \operatorname{Hom}_{A}\left(\mathrm{H}_{2}(R, A, A), A\right)=0$; the latter because ${ }_{v} \mathrm{H}_{2}(R, A, A) \hookrightarrow S_{2}(I) v_{v}=0$ for $v<6$ and the socle degree of $A$ is 4 . Since ${ }_{0} \operatorname{hom}_{A}\left(I / I^{2}, A\right)=20$ by Macaulay 2, we get by Theorem 4.1 that codim $\operatorname{GradAlg}(H) \overline{W(\underline{b} ; \underline{a})}=4$.

(ii) Again $R=k\left[x_{0}, x_{1}, x_{2}\right]$, but now $\mathcal{A}$ is a general $3 \times 5$ matrix with quadratic (resp. linear) entries in the first (resp. second and third) row. The vanishing of all $3 \times 3$ minors defines an artinian ring with $h$-vector $(1,3,6,10,5,1)$. Using Macaulay 2 one verifies that all conditions of Theorem $3.5(\mathrm{i})$ hold, and we get that $\overline{W_{s}(\underline{b} ; \underline{a})}$ is an irreducible subset of $\operatorname{Grad} \operatorname{Alg}(H)$ of dimension $\lambda_{3}+K_{3}=25$. Since ${ }_{0} \operatorname{Ext}_{A}^{1}\left(I / I^{2}, A\right)=0$ by Macaulay 2 and ${ }_{v} \mathrm{H}_{2}(R, A, A) \hookrightarrow S_{2}(I)_{v}=0$ for $v<8$, we get ${ }_{0} \operatorname{Hom}_{A}\left(\mathrm{H}_{2}(R, A, A), A\right)=0$, whence $A$ is unobstructed by (2.2). Then Theorem 4.1 and ${ }_{0} \operatorname{hom}_{A}\left(I / I^{2}, A\right)=40$ (by Macaulay 2) imply that $\operatorname{codim}_{\operatorname{GradAlg}(H)} \overline{W(\underline{b} ; \underline{a})}=15$.

Remark 4.5. Another possible way of finding the dimension of $\overline{W_{s}(\underline{b} ; \underline{a})}$ and its codimension in $\operatorname{GradAlg}(H)$, as well as determining whether $\operatorname{GradAlg}\left(H_{A}\right)$ is smooth at $(A) \in \overline{W_{s}(\underline{b} ; \underline{a})} \subset \operatorname{GradAlg}\left(H_{A}\right)$, is to consider some closely related and well-understood family of $\operatorname{GradAlg}\left(H_{B}\right)$ containing a point $(B)$ for which there is a surjection $B \rightarrow A$ of $k$-algebras whose Hilbert functions satisfy $H_{B}(v)=H_{A}(v)$ for every $v \leq s$ and $s$ sufficiently large. Such a comparison is done in [29] when $\operatorname{dim} A \geq 1$, using $B=R / I_{t}(\mathcal{B})$, $A=R / I_{t}(\mathcal{A})$ and $\mathcal{A}=[\mathcal{B}, v], v$ a column, cf. Example 4.2. In [28] we explore this approach more generally, e.g. see [28, Thm. 29] for a result where $A$ is artinian in $B \rightarrow A$.

In the case $B=R / I_{t}(\mathcal{B})$ where $\mathcal{A}=[\mathcal{B}, v]$ and $v$ a column, we generalize in Sec. 6 of this paper several results from [29], see in particular Theorem [6.6] where $\operatorname{dim} A=1$. It is possible to extend this theorem to $A$ artinian by increasing the set of assumptions in Theorem [6.6, but since this theorem is already quite complicated as stated, we think 
Theorem 4.1 is much simpler. It is also an option to replace $B=R / I_{t}(\mathcal{B})$ by an artinian Gorenstein quotient $A_{g}$ of $B$ such that $B \rightarrow A$ factors via $A_{g} \rightarrow A$ and, for $c=3$, use either [26, Thm. 2.3] or [8, Sec. 5] to compute e.g. $\operatorname{dim}_{\left(A_{g}\right)} \operatorname{GradAlg}\left(H_{A_{g}}\right)$. Applying, however, [27, Thm. 1] to $B \rightarrow A_{g}$ which states that $\operatorname{GradAlg}\left(H_{A_{g}}\right)$ and $\operatorname{GradAlg}\left(H_{B}\right)$ are much related, especially when $c=3$ by [27, Example 28], this latter approach is quite similar (concerning assumptions to be fulfilled) to the approach using $R / I_{t}(\mathcal{B}) \rightarrow A$.

We have the following generalization of [31, Theorem 5.8] in which the good determinantal assumption $\left(\operatorname{depth}_{I_{t-1}(\mathcal{A}) A} A \geq 1\right)$ is weakened, allowing $A$ to be artinian. Note that we below may replace $\operatorname{Grad} \operatorname{Alg}(H)$ with $\operatorname{Hilb}^{p}\left(\mathbb{P}^{n}\right)$ if $\operatorname{dim} A \geq 2$, cf. (2.1).

Theorem 4.6. Let $A=R / I, I=I_{t}(\mathcal{A})$ be a standard determinantal k-algebra, i.e. $(A) \in W_{s}(\underline{b} ; \underline{a})$, let $M=\operatorname{coker} \varphi^{*}$ and suppose ${ }_{0} \operatorname{Hom}_{A}(M, M) \simeq k$ and ${ }_{0} \operatorname{Ext}_{A}^{i}(M, M)=0$ for $i=1$ and 2 . Then $\operatorname{GradAlg}(H)$ is smooth at $(A)$ and

$$
\operatorname{dim}_{(A)} \operatorname{GradAlg}(H)=\lambda_{c}+K_{3}+K_{4}+\ldots+K_{c},
$$

(cf. Remark [.6)). Moreover $\overline{W_{s}(\underline{b} ; \underline{a})} \subset \operatorname{GradAlg}(H)$ is an irreducible component, and every deformation of $A$ comes from deforming $\mathcal{A}$. In particular these conclusions hold if $\operatorname{dim} A \geq 4+\operatorname{dim} R / I_{t-1}(\mathcal{A})$, or if $\operatorname{dim} A \geq 3$ and $\operatorname{dim} R / I_{t-1}(\mathcal{B})=0$ where $\mathcal{B}$ is obtained from $\mathcal{A}$ by deleting some column of $\mathcal{A}$ (e.g. if $\operatorname{dim} A \geq 3, a_{i-\min (3, t)} \geq b_{i}$ for $\min (3, t) \leq i \leq t$ and $A$ is general).

Proof. The first two sentences of conclusions follow from Theorem 3.5 and Lemma 2.10. Moreover since $\operatorname{depth}_{I_{t-1}(\mathcal{A}) A} A=\operatorname{dim} A-\operatorname{dim} R / I_{t-1}(\mathcal{A})$, the final sentence follows directly from [31, Theorem 5.8], and the parenthesis from Remark 2.5.

The condition ${ }_{0} \operatorname{Ext}_{A}^{2}(M, M)=0$ of Theorem 4.6 seems harder to satisfy for artinian rings, but it holds quite often if $\mathcal{A}$ is not too close to the linear case. Here is an example:

Example 4.7. (determinantal artinian quotients of $R=k\left[x_{0}, x_{1}, x_{2}\right]$, using Theorem 4.6)

(i) Let $\mathcal{A}=[\mathcal{B}, v]$ be a general $2 \times 4$ matrix with linear (resp. cubic) entries in the first and second (resp. third) column and where the entries of $v$ are polynomials of the same degree $m, m \geq 3$. The degree matrix of $\mathcal{A}$ is $\left(\begin{array}{llll}1 & 1 & 3 & \mathrm{~m} \\ 1 & 1 & 3 & \mathrm{~m}\end{array}\right)$. The vanishing of all $2 \times 2$ minors defines an artinian ring with $h$-vector $(1,3,5,7,7, . ., 7,5,3)$, where the number of $7 s$ is $m-2$. For $m=3$ and $m \geq 5$ one verifies that all conditions of Theorem 3.5(ii), i.e. the first conditions of Theorem 4.6 hold, and it follows that $\overline{W_{s}(\underline{b} ; \underline{a})}$ is a generically smooth irreducible component of $\operatorname{GradAlg}(H)$ of dimension $\lambda_{3}+K_{3}=18$ (resp. 17) for $m \geq 5$ (resp. $m=3$ ). The computations for the ${ }_{0}$ Ext $^{i}$-groups above are verified by Macaulay 2 for $m \leq 10$ (cf. Remark 4.3), but their dimensions hold also for $m>10$. One also verifies that ${ }_{0} \operatorname{ext}_{A}^{1}\left(I / I^{2}, A\right)=2$ for $6 \leq m \leq 10$, so both the generic smoothness (cf. (2.2)) along $\overline{W_{s}(\underline{b} ; \underline{a})}$ and the conclusion that $\overline{W_{s}(\underline{b} ; \underline{a})}$ is an irreducible component of $\operatorname{GradAlg}(H)$ would be hard to see without using Theorem 4.6.

(ii) Let $\mathcal{A}$ be a general $2 \times 4$ matrix with degree matrix $\left(\begin{array}{llll}3 & 3 & 3 & 3 \\ 1 & 1 & 1 & 1\end{array}\right)$. The vanishing of all $2 \times 2$ minors defines an artinian ring with $h$-vector $(1,3,6,10,9,7,3,1)$. Using Macaulay 2 one verifies that all conditions of Theorem 3.5 (ii) hold, (i.e. also ${ }_{0} \operatorname{Ext}_{A}^{2}(M, M)=0$ ), and we get that $\overline{W_{s}(\underline{b} ; \underline{a})}$ is a generically smooth irreducible component of $\operatorname{GradAlg}(H)$ of dimension $\lambda_{3}+K_{3}=29$. In this example ${ }_{0} \operatorname{Ext}_{A}^{1}\left(I / I^{2}, A\right) \neq 0$ and ${ }_{0} \operatorname{hom}_{A}\left(I / I^{2}, A\right)=29$, 
so it is not straightforward to see that $A$ is unobstructed, but it is, due to Theorem 4.6 which also contains additional information.

As indicated it seems that the only artinian determinantal rings $A=R / I_{t}(\mathcal{A})$ in the case $a_{0}>b_{t}$ that do not satisfy the conditions of Theorem 3.5)(i) are those with a matrix $\mathcal{A}$ that is linear except in one column $v \in R_{m}^{\oplus t}$ where the degree is $m \geq 2$. Moreover if $m \geq 3$ we may even have

$$
{ }_{0} \operatorname{Hom}_{A}(M, M) \simeq k, \quad{ }_{0} \operatorname{Ext}_{A}^{1}(M, M) \neq 0 \text { and }{ }_{0} \operatorname{Ext}_{A}^{2}(M, M)=0,
$$

and the deformation functors of these rings are fully determined by our next Theorem 4.8 , For artinian linear determinantal rings (i.e. $m=1$ above) all conditions of Theorem 3.5(ii) seem to hold and even more, they are rigid in several senses: ${ }_{0} \operatorname{Hom}_{R}(I, A)=0$, as well as ${ }_{0} \operatorname{Ext}_{R}^{1}(M, M)=0$, whence the linear case may be not so interesting. Recalling (3.4), i.e. $\lambda:=\operatorname{dim}{ }_{0} \operatorname{Ext}_{R}^{1}(M, M)=\lambda_{c}+K_{3}+K_{4}+\ldots+K_{c}$ and Remark 2.6, we get

Theorem 4.8. Let $A=R / I, I=I_{t}(\mathcal{A})$ be a standard determinantal k-algebra, let $M=$ coker $\varphi^{*}$ and suppose ${ }_{0} \operatorname{Hom}_{A}(M, M) \simeq k$. If the map ${ }_{0} \operatorname{Ext}_{A}^{2}(M, M) \rightarrow{ }_{0} \operatorname{Ext}_{R}^{2}(M, M)$ of (3.1) is injective, or weaker, if the tangent map ${ }_{0} \operatorname{Ext}_{R}^{1}(M, M) \rightarrow{ }_{0} \operatorname{Hom}_{R}(I, A)$ of the natural morphism of functors $e_{M}: \operatorname{Def}_{M / R} \rightarrow \operatorname{Def}_{A / R}$ is surjective, then $e_{M}$ is smooth. Moreover $\operatorname{Def}_{M / A}$ is smooth and its pro-representing object $H(M / A)$ satisfies

$$
\operatorname{dim} H(M / A)=\operatorname{dim}_{0} \operatorname{Ext}_{A}^{1}(M, M)=\lambda-\operatorname{dim} W_{s}(\underline{b} ; \underline{a}) .
$$

Furthermore $\operatorname{Def}_{A \in W_{s}(\underline{b} ; \underline{a})}=\operatorname{Def}_{A / R}, \operatorname{Def}_{A / R}$ is smooth, $\overline{W_{s}(\underline{b} ; \underline{a})} \subset \operatorname{GradAlg}(H)$ is an irreducible component of dimension $\lambda-\operatorname{dim}{ }_{0} \operatorname{Ext}_{A}^{1}(M, M)$ and every deformation of $A$ comes from deforming $\mathcal{A}$. In particular these conclusions hold if $\operatorname{dim} A \geq 3+\min \left\{\operatorname{dim} R / I_{t-1}(\mathcal{A})+\right.$ $\left.1, \operatorname{dim} R / I_{t-1}(\mathcal{B})\right\}$ where $\mathcal{B}$ is obtained from $\mathcal{A}$ by deleting some column of $\mathcal{A}$ (e.g. if $\operatorname{dim} A \geq 3, a_{i-\min (3, t)} \geq b_{i}$ for $\min (3, t) \leq i \leq t$ and $A$ is general).

Remark 4.9. We may consider $\operatorname{Def}_{M / A}$ as a fiber functor of $e_{M}: \operatorname{Def}_{M / R} \rightarrow \operatorname{Def}_{A / R}$, using trivial deformations of $A$. For a description of the obstruction maps of $\operatorname{Def}_{M / A}$ for arbitrary $M$, see [40, 23]. Note that the assumption ${ }_{0} \operatorname{Hom}_{A}(M, M) \simeq k$ implies the pro-representability of both $\operatorname{Def}_{M / A}$ and $\operatorname{Def}_{M / R}$, cf. end of proof of Theorem 3.4.

Proof. Firstly we observe that the composition of the natural maps:

$$
{ }_{0} \operatorname{Ext}_{R}^{1}(M, M) \stackrel{e_{M}(D)}{\longrightarrow}{ }_{0} \operatorname{Hom}_{R}(I, A) \hookrightarrow{ }_{0} \operatorname{Hom}_{R}\left(I, \operatorname{Hom}_{A}(M, M)\right) \simeq\left(E_{2}^{0,1}\right)_{0}
$$

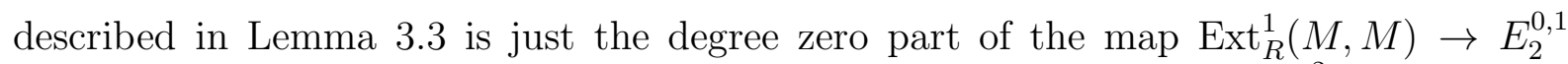
appearing in (3.1). This composition is surjective if and only if the ${ }_{0} \mathrm{Ext}_{R}^{2}$-map of Theorem 4.8 is injective by (3.1). Since $A \rightarrow \operatorname{Hom}_{A}(M, M)$ is injective, it follows that the injectivity of this ${ }_{0} \operatorname{Ext}_{R}^{2}$-map is equivalent to the surjectivity of $e_{M}(D):{ }_{0} \operatorname{Ext}_{R}{ }_{R}(M, M) \rightarrow$ ${ }_{0} \operatorname{Hom}_{R}(I, A)$ and ${ }_{0} \operatorname{Hom}_{R}(I, A) \simeq{ }_{0} \operatorname{Hom}_{R}\left(I, \operatorname{Hom}_{A}(M, M)\right)$ being bijective.

Let $T \rightarrow S$ be a small artinian surjection with kernel $\mathfrak{a}$. To prove the (formal) smoothness of $e_{M}: \operatorname{Def}_{M / R} \rightarrow \operatorname{Def}_{A / R}$, we must by definition show that the induced map

$$
\operatorname{Def}_{M / R}(T) \rightarrow \operatorname{Def}_{M / R}(S) \times_{\operatorname{Def}_{A / R}(S)} \operatorname{Def}_{A / R}(T)
$$

is surjective. Let $M_{S}$ be an arbitrary fixed deformation of $M$ to $S$ inducing $R_{S} / I_{S} \in$ $\operatorname{Def}_{A / R}(S)$, let $R_{T} / I_{T}$ be a deformation of $R_{S} / I_{S}$ to $T$ and let $e_{M_{S}}$ be the functor that 
takes a deformation $M_{T}=\operatorname{coker}\left(\varphi^{*}\right)$ of $M_{S}$ onto the deformation of $R_{S} / I_{S}$ defined by the maximal minors of a matrix representing $\varphi$. Note that since $M$ is unobstructed, there exists a deformation $M_{T}^{\prime}$ of $M_{S}$ to $T$ inducing a deformation $R_{T} / I_{T}^{\prime}$ of $R_{S} / I_{S}$. The difference " $R_{T} / I_{T}-R_{T} / I_{T}^{\prime}$ " sits in ${ }_{0} \operatorname{Hom}_{R}(I, A) \otimes_{k} \mathfrak{a}$ and since $e_{M_{S}}(T) \otimes_{T} i d_{\mathfrak{a}}$ is up to isomorphism equal to the surjective map $e_{M}(D) \otimes_{k} \mathfrak{a}$ as one may see by the arguments described in the first paragraph of the proof of Lemma 3.3, $e_{M_{S}}(T) \otimes_{T} i d_{\mathfrak{a}}$ is surjective. Hence there exists an element in ${ }_{0} \operatorname{Ext}_{R}^{1}(M, M) \otimes_{k} \mathfrak{a}$ that we can "add" to $M_{T}^{\prime}$ to get a deformation $M_{T}$ of $M_{S}$ that induces $R_{T} / I_{T}$, i.e. $e_{M}$ is smooth. It follows that $\operatorname{Def}_{A / R}$ is smooth. We also get that $\operatorname{Def}_{A \in W_{s}(\underline{b} ; \underline{a})}(T) \hookrightarrow \operatorname{Def}_{A / R}(T)$ is surjective for any $T \in \mathrm{ob}(\underline{\ell})$, and injective by Definition 3.2, whence an equality, and since $\operatorname{Def}_{M / A}$ is a fiber functor of $\operatorname{Def}_{M / R} \rightarrow \operatorname{Def}_{A / R}$, it follows that $\operatorname{Def}_{M / A}$ is smooth. Hence $\operatorname{dim} H(M / A)=\operatorname{dim}{ }_{0} \operatorname{Ext}_{A}^{1}(M, M)$, and we get the displayed dimension formulas of Theorem 4.8 from (3.1) and the surjectivity of ${ }_{0} \operatorname{Ext}_{R}^{1}(M, M) \rightarrow{ }_{0} \operatorname{Hom}_{R}(I, A)$ provided $\operatorname{dim} W_{s}(\underline{b} ; \underline{a})={ }_{0} \operatorname{hom}_{R}(I, A)$.

If we compare $\operatorname{Def}_{A \in W_{s}(\underline{b} ; \underline{a})}=\operatorname{Def}_{A / R}$ with Definition 3.2 we get that every deformation $A_{T}$ of $A$ to an artinian $T$ comes from deforming $\mathcal{A}$. To see that we may replace "an artinian $T$ " by " $T$ a local ring" in this statement (cf. Definition 2.9) we pick $d:={ }_{0} \operatorname{hom}_{R}(I, A)$ elements of ${ }_{0} \operatorname{Ext}_{R}^{1}(M, M)$ that map to linearly independent elements of ${ }_{0} \operatorname{Hom}_{R}(I, A)$ via the tangent map ${ }_{0} \operatorname{Ext}_{R}^{1}(M, M) \rightarrow{ }_{0} \operatorname{Hom}_{R}(I, A)$ of $e_{M}$, and we let $\eta_{1}, \ldots, \eta_{d} \in{ }_{0} \operatorname{Hom}\left(G^{*}, F^{*}\right)$ with presentation matrices $\mathcal{A}_{1}, \ldots, \mathcal{A}_{d}$ correspond to the $d$ elements we picked in ${ }_{0} \operatorname{Ext}_{R}^{1}(M, M)$, cf. the beginning of the proof of Lemma 3.3 for a similar set-up. Let $\mathcal{A}_{S}:=\mathcal{A}+s_{1} \mathcal{A}_{1}+\ldots+s_{d} \mathcal{A}_{d}$ and $S:=k\left[s_{1}, \ldots, s_{d}\right]_{\left(s_{1}, \ldots, s_{d}\right)}$ where $k\left[s_{1}, \ldots, s_{d}\right]$ is a polynomial ring, and let $O:=\mathcal{O}_{\operatorname{GradAlg}(H),(A)}$ be the local ring of $\operatorname{GradAlg}(H)$ at the $k$-point $(A) \in \operatorname{GradAlg}(H), k=\bar{k}$. Then the algebraic family $A_{S}:=R_{S} / I_{t}\left(\mathcal{A}_{S}\right)$ is $S$-flat by Lemma 2.8 and e.g. using the explicit description of the pro-representing object $H(A / R)$ of $\operatorname{Def}_{A / R}$ appearing in the proof of Thm. 4.2.4 of [39] to see that any deformation of $A_{S} \otimes_{S} S /\left(s_{1}, \ldots, s_{d}\right)^{2}$ to $H(A / R)$ may serve as a versal lifting, we get that $H(A / R)$ equals $S$ with "universal object" $A_{S}$ (up to completion, but notice that the versal lifting is defined by polynomials, not power series, in $s_{1}, \ldots, s_{d}$ ). Hence, by the universal property of the representable functor that corresponds to $\operatorname{GradAlg}(H)$, there is a morphism $O \rightarrow S$ whose completions are isomorphic because both pro-represents $\operatorname{Def}_{A / R}$ on $\ell([48$, $(2,1)$ and (2.8)]). Thus $O \rightarrow S$ is injective, and since $O / \mathfrak{m}_{O}^{n} \simeq S / \mathfrak{m}_{S}^{n}$ for every $n$, it follows that $O \rightarrow S$ is an isomorphism, having $A_{O}:=A_{S} \otimes_{S} O$ as the pullback (considered as scheme) of the universal object of $\operatorname{GradAlg}(H)$ to $\operatorname{Spec}(O)$. This shows that "every deformation of $A$ comes from deforming $\mathcal{A}$ " because the universal property implies that all deformations are given by pullback to $\operatorname{Spec}(T)$ (i.e. by taking tensor product of $O \rightarrow A_{O}$ via $O \rightarrow T$ ). Then Lemma 2.10 implies that $\overline{W_{s}(\underline{b} ; \underline{a})} \subset \operatorname{GradAlg}(H)$ is a generically smooth irreducible component of dimension $d$, and in particular we have $\operatorname{dim} W_{s}(\underline{b} ; \underline{a})={ }_{0} \operatorname{hom}_{R}(I, A)$.

The argument for the statement in the final sentence is as in the proof of Theorem 4.6 provided $\operatorname{dim} A \geq 4+\operatorname{dim} R / I_{t-1}(\mathcal{A})$. If, however, $\operatorname{dim} A \geq 3+\operatorname{dim} R / I_{t-1}(\mathcal{B})$ then $\operatorname{depth}_{I_{t-1}(\mathcal{B})} R / I_{t}(\mathcal{B}) \geq 4$ and we get ${ }_{0} \operatorname{Ext}_{A}^{2}(M, M)=0$ by [31, Thm. 4.5] and we are done.

As a corollary to the $3^{\text {rd }}$ paragraph of the proof, we get that if every graded deformation of $A$ to any local artinian $k$-algebra comes from deforming $\mathcal{A}$, then every deformation of $A$ comes from deforming $\mathcal{A}$ (see Definition 2.9). But the proof implies more: 
Corollary 4.10. Let $A=R / I_{t}(\mathcal{A})$ be a standard determinantal $k$-algebra. If every graded deformation $A_{D}$ of $A$ to the dual numbers $D:=k[\epsilon] /\left(\epsilon^{2}\right)$ comes from deforming $\mathcal{A}$, or equivalently, if the tangent map of the natural morphism $e_{M}: \operatorname{Def}_{M / R} \rightarrow \operatorname{Def}_{A / R}$,

$$
e_{M}(D): \operatorname{Def}_{M / R}(D)={ }_{0} \operatorname{Ext}_{R}^{1}(M, M) \rightarrow \operatorname{Def}_{A / R}(D)={ }_{0} \operatorname{Hom}_{R}(I, A),
$$

is surjective, then every deformation of $A$ comes from deforming $\mathcal{A}$.

Proof. Since every graded deformation of $M$ comes from deforming $\mathcal{A}$ by Lemma 2.8, it is clear that "every graded deformation of $A$ to $D$ comes from deforming $\mathcal{A}$ " is equivalent to the surjectivity of the tangent map $e_{M}(D)$. Then we get what we want from the proof appearing in the $3^{\text {rd }}$ paragraph of Theorem 4.8 since that part of the proof only requires the surjectivity of $e_{M}(D)$ and not the condition ${ }_{0} \operatorname{Hom}_{A}(M, M) \simeq k$.

Let us consider two particular cases of artinian rings:

Example 4.11. (determinantal artinian quotients of $R$, using Theorem 4.8)

(i) Let $R=k\left[x_{0}, x_{1}, x_{2}\right]$ and let $\mathcal{A}=[\mathcal{B}, v]$ be a general $2 \times 4$ matrix where $\mathcal{B}$ is linear and the entries of $v$ are polynomials of the same degree $m \geq 2$. The vanishing of all $2 \times 2$ minors defines an artinian ring with $h$-vector $(1,3,3, . ., 3)$ where the number of $3 \mathrm{~s}$ is $\mathrm{m}$. Using Macaulay 2 for $3 \leq m \leq 10$ one verifies (cf. Remark 4.3) that all conditions of Theorem 4.8 hold and that ${ }_{0} \operatorname{ext}_{A}^{1}(M, M)=2$, and it follows that $\overline{W_{s}(\underline{b} ; \underline{a})}$ is a generically smooth irreducible component of $\operatorname{GradAlg}(H)$ of dimension $\lambda_{3}+K_{3}-{ }_{0} \operatorname{ext}_{A}^{1}(M, M)=6$ (true also for $m>10$ ). If $m=2$, Theorem 4.8 does not apply because ${ }_{0} \operatorname{Ext}_{A}^{2}(M, M) \neq 0$. In fact one may verify (Macaulay 2) that all inclusions of (3.5) are strict, i.e. nonisomorphisms.

(ii) Let $R=k\left[x_{0}, x_{1}, x_{2}, x_{3}\right]$ and let $\mathcal{A}=[\mathcal{B}, v]$ be a general $2 \times 5$ matrix where $\mathcal{B}$ is linear and the entries of $v$ are polynomials of the same degree $m \geq 2$. The vanishing of all $2 \times 2$ minors defines an artinian ring with $h$-vector $(1,4,4 . .,, 4)$, where the number of $4 s$ is $m$. Using Macaulay 2 for $3 \leq m \leq 10$ one verifies that all conditions of Theorem 4.8 hold and that ${ }_{0} \operatorname{ext}_{A}^{1}(M, M)=4$. By Proposition 2.4 and (2.16), $K_{3}=0, K_{4}=4$ and $\lambda_{4}=12$, and it follows that $\overline{W_{s}(\underline{b} ; \underline{a})}$ is a generically smooth irreducible component of $\operatorname{Grad} \operatorname{Alg}(H)$ of dimension $\lambda_{4}+K_{3}+K_{4}-{ }_{0} \operatorname{ext}_{A}^{1}(M, M)=12$ (true also for $m>10$ ).

Remark 4.12. Comparing Theorem 4.8 with Theorem 4.6 we see that the main condition for $\overline{W_{s}(\underline{b} ; \underline{a})}$ to be an irreducible component of $\operatorname{GradAlg}\left(H_{A}\right)$ is that ${ }_{0} \operatorname{Ext}_{A}^{2}(M, M)$ (or $\operatorname{ext}^{2}(M, M)$, cf. Theorem 4.1) vanishes; the condition ${ }_{0} \operatorname{Ext}_{A}^{1}(M, M)=0$ of Theorem 4.6 is in this regard less important. An interesting observation to make to all examples of this section is that ghost terms in the minimal resolution of $A$ appear precisely when ${ }_{0} \operatorname{Ext}_{A}^{2}(M, M) \neq 0$. Indeed for all $A$ in Examples 4.2 and 4.4 we have ${ }_{0} \operatorname{Ext}_{A}^{2}(M, M) \neq 0$ (Macaulay 2) as well as ghost terms in the minimal resolution of $A$ while we in Examples 4.7 and 4.11 where $\overline{W_{s}(\underline{b} ; \underline{a})}$ is a component due to ${ }_{0} \operatorname{Ext}_{A}^{2}(M, M)=0$, have no ghost terms in the minimal resolution of $A$. So one may wonder if there is a connection between ${ }_{0} \operatorname{Ext}_{A}^{2}(M, M) \neq 0$ and the existence of ghost terms for small $c$ in the artinian case (and maybe also when $\operatorname{dim} A \leq 1$, cf. Example 4.13, but not when $\operatorname{dim} A \geq 2$, cf. Example 5.5). Indeed we devote the next section to a study of ghost terms in the Eagon-Northcott resolution (2.5). 
For determinantal zero-schemes we showed in [29] that the dimension formula (2.15) fails when (and only when?) $\mathcal{A}$ is a linear matrix consisting of two rows, cf. Remark 2.7(ii). In our next example we use Theorem 4.8 to treat this case.

Example 4.13. (determinantal quotients of $R=k\left[x_{0}, x_{1}, \cdots, x_{c}\right]$ of dimension one)

Let $\mathcal{A}$ be a general $2 \times(c+1)$ matrix of linear entries. The vanishing all $2 \times 2$ minors defines a reduced scheme $X=\operatorname{Proj}(A)$ of $c+1$ general points in $\mathbb{P}^{c}$. Since we may suppose $A$ is good determinantal, $\operatorname{Hom}_{A}(M, M) \simeq A$ by [33, Lem. 3.2], and we have ${ }_{0} \operatorname{ext}_{R}^{1}(M, M)=c(c+1)+c-2$ by Theorem 3.4. In the range $3 \leq c \leq 10$ we use Macaulay 2 to see ${ }_{0} \operatorname{ext}_{A}^{1}(M, M)=c-2$ and ${ }_{0} \operatorname{Ext}_{A}^{2}(M, M)=0$. For $c \leq 10$ we get from Theorem 4.8 that $\overline{W(0,0 ; 1,1, \ldots, 1)}$ is a generically smooth irreducible component of $\operatorname{Grad} A \lg (H)$ of dimension $c(c+1)$ and that every deformation of $A$ comes from deforming $\mathcal{A}$.

It is possible to describe $\overline{W(0,0 ; 1,1, \ldots, 1)}$ for all $c \geq 2$ without using Macaulay 2. Indeed it is well known that there exists a generically smooth component of the usual Hilbert scheme $\operatorname{Hilb}^{p}\left(\mathbb{P}^{c}\right)$, of dimension $c(c+1)$, containing an open subset parametrizing $c+1$ reduced points in $\mathbb{P}^{c}$. Since the Hilbert function of $X$ is $\left(\operatorname{dim} A_{i}\right)=(1, c+1, c+1, \ldots)$, we get $H^{1}\left(\mathcal{I}_{X}(v)\right) \simeq{ }_{v} H_{\mathfrak{m}}^{1}(A)=0$ for $v \geq 1$ and hence ${ }_{0} \operatorname{Hom}_{R}\left(I_{X}, H_{\mathfrak{m}}^{1}(A)\right)=0 . \operatorname{GradAlg}(H)$ is therefore isomorphic to $\operatorname{Hilb}^{p}\left(\mathbb{P}^{c}\right)$ at $(X)$ by $(2.1)$, and it follows that $\overline{W(0,0 ; 1,1, \ldots, 1)}$ is a generically smooth irreducible component of $\operatorname{GradAlg}(H)$ of dimension $c(c+1)$.

Theorem 4.8 admits a nice consequence concerning glicciness. Indeed since glicciness is not necessarily an open property, the following result may be useful.

Corollary 4.14. Let $X=\operatorname{Proj}(A), I_{X}=I_{t}(\mathcal{A})$, be a standard determinantal scheme and suppose that ${ }_{0} \operatorname{Hom}_{R}\left(I_{X}, H_{\mathfrak{m}}^{1}(A)\right)=0$ and that the $\operatorname{map}{ }_{0} \operatorname{Ext}_{A}^{2}(M, M) \rightarrow{ }_{0} \operatorname{Ext}_{R}^{2}(M, M)$ of (3.1) is injective. Then the Hilbert scheme $\operatorname{Hilb}^{p}\left(\mathbb{P}^{n}\right)$ is smooth at $(X)$ and $(X)$ belongs to a unique irreducible component of $\operatorname{Hilb}^{p}\left(\mathbb{P}^{n}\right)$ whose general element $\tilde{X} \subset \mathbb{P}^{n}$ is glicci. In particular this conclusion holds if $\operatorname{dim} X \geq 2+\min \left\{\operatorname{dim} R / I_{t-1}(\mathcal{A})+1, \operatorname{dim} R / I_{t-1}(\mathcal{B})\right\}$ where $\mathcal{B}$ is obtained from $\mathcal{A}$ by deleting some column of $\mathcal{A}$.

Proof. Indeed $\tilde{X}$ is standard determinantal by Theorem 4.8 and (2.1) and since standard determinantal schemes are glicci by [32, Thm. 3.6], we are done.

Finally we generalize [31, Thm. 5.16] which is about codimension-2 determinantal quotients of an ACM scheme, to cover the artinian case. Below $\bar{R}$ is a Cohen-Macaulay quotient of a polynomial ring (i.e. $\operatorname{Proj}(\bar{R}) \subset \mathbb{P}_{k}^{N}$ is $\mathrm{ACM}$ ) and $A$ is a standard determinantal quotient of $\bar{R}$, but notice that it is not necessarily a determinantal quotient of $k\left[x_{0}, \cdots, x_{N}\right]$. Moreover letting $\underline{b}, \underline{a}$ be as in (2.4) and $(A) \in W_{s}(\underline{b} ; \underline{a})$ observe that, for $\operatorname{dim} A \geq 2$, we may replace $\operatorname{GradAlg}^{H}(\bar{R})$ by $\operatorname{Hilb}^{p}(\operatorname{Proj}(\bar{R}))$ since (2.1) extends to hold in this generality by [25, Thm. 3.6, Rem. 3.7]. For the irreducibility and dimension of $\overline{W_{s}(\underline{b} ; \underline{a})}$ in the case $\bar{R}=k\left[x_{0}, x_{1}\right]$, see also [19, Thms. 2.9, 2.12, 3.13].

Theorem 4.15. Let $P=\operatorname{Proj}(\bar{R}) \subset \mathbb{P}_{k}^{N}$ be an $A C M$ scheme where $k$ is any field and let $X=\operatorname{Proj}(A) \subset P, A=R / I_{t}(\mathcal{A})$, be any standard determinantal scheme of codimension 2 in $P$. Then $\operatorname{GradAlg}^{H}(\bar{R})$ is smooth at $(A)$ and $\operatorname{dim}_{(A)} \operatorname{GradAlg}^{H}(\bar{R})=\lambda(\bar{R})_{2}$ where

$$
\lambda(\bar{R})_{2}:=\sum_{i, j} \operatorname{dim} \bar{R}_{\left(a_{j}-b_{i}\right)}+\sum_{i, j} \operatorname{dim} \bar{R}_{\left(b_{i}-a_{j}\right)}-\sum_{i, j} \operatorname{dim} \bar{R}_{\left(a_{i}-a_{j}\right)}-\sum_{i, j} \operatorname{dim} \bar{R}_{\left(b_{i}-b_{j}\right)}+1 .
$$


Moreover every deformation of $A$ comes from deforming $\mathcal{A}$. In particular if $k=\bar{k}$, then $\operatorname{GradAlg}^{H}(\bar{R})$ is smooth along $W_{s}(\underline{b} ; \underline{a})$ and the closure $\overline{W_{s}(\underline{b} ; \underline{a})}$ in $\operatorname{GradAlg}^{H}(\bar{R})$ is an irreducible component of dimension $\lambda(\bar{R})_{2}$.

Proof. Since $M$ is a twist of the canonical module of $A$ when $c=2$, it is well known that ${ }_{0} \operatorname{Ext}_{A}^{i}(M, M)=0$ for $i>0$ and $\operatorname{Hom}_{A}(M, M) \simeq A$. We get $\operatorname{Def}_{M / \bar{R}} \simeq \operatorname{Def}_{A / \bar{R}}$ and every statement of the theorem from Theorem 3.5, Lemma 2.10 and Remark 3.6, except the displayed formula. Using, however, the last conclusion of Theorem 3.4, we easily prove the displayed formula, cf. (2.6). Finally note that we have included the assumption $k=\bar{k}$ in the statements of $W_{s}(\underline{b} ; \underline{a})$ for the only reason that $W_{s}(\underline{b} ; \underline{a})$ is by definition a certain locus (of $k$-points) of $\operatorname{GradAlg}^{H}(\bar{R})$.

So there are no singular points of $\operatorname{GradAlg}^{H}(\bar{R})$ at $(A) \in W_{s}(\underline{b} ; \underline{a})$ when $c=2$ while singular points of $\operatorname{GradAlg}^{H}(\bar{R})$ for $c>2$ are quite common (see [34, Rem. 3.6] and [49]).

\section{GHOST TERMS}

Let $N$ be a graded $R$-module with a minimal $R$-free resolution $0 \rightarrow P_{\bullet} \rightarrow N$. By a ghost term of $P_{\bullet}$ we mean a direct free summand that appears in consecutive terms of $P_{\boldsymbol{\bullet}}$. As in the minimal resolution conjecture (41, 46], and see [43, 42, 45] and its references for other contributions), one expects that a general element $R / I$ of $\operatorname{GradAlg}(H)$ contains "as few ghost terms as possible", while ghost terms for special elements of $\operatorname{Grad} \operatorname{Alg}(H)$ are more common. Indeed the semicontinuity of graded Betti numbers imply that these numbers decrease under generization (deformation to a more general element), but there may still be ghost terms left in the minimal free resolution of the general element of $\operatorname{Grad} \operatorname{Alg}(H)$. In this section we shall see that some ghost terms of a determinantal ring $R / I$ easily disappear under generizations while other ghost terms do not. Letting $\underline{a}_{\hat{i}}=a_{0}, \ldots a_{i-1}, a_{i+1} \ldots, a_{t+c-2}$ and $\underline{b}_{\hat{i}}=b_{1}, \ldots b_{i-1}, b_{i+1} \ldots, b_{t}$ we have the following result (allowing $t=2$, in which case $W_{s}\left(\underline{b}_{i} ; \underline{a}_{\hat{j}}\right)$ consists of complete intersections of $R$ ).

Proposition 5.1. Suppose $a_{j}=b_{i}$ for some $j \in\{0, t+c-2\}$ and $i \in\{1, t\}$. Then we have an inclusion $W_{s}\left(\underline{b}_{i} ; \underline{a}_{\hat{j}}\right) \subset W_{s}(\underline{b} ; \underline{a})$ of open irreducible subsets of $\operatorname{Grad} \operatorname{Alg}(H)$. Moreover if $W_{s}(\underline{b} ; \underline{a}) \backslash W_{s}\left(\underline{b}_{\hat{i}} ; \underline{a}_{\hat{j}}\right) \neq \emptyset$, then every $R / I$ of $W_{s}(\underline{b} ; \underline{a}) \backslash W_{s}\left(\underline{b}_{\hat{i}} ; \underline{a}_{\hat{j}}\right)$ admits a generization $\left(R / I_{g}\right) \in W_{s}\left(\underline{b}_{i} ; \underline{a}_{\hat{j}}\right)$ removing exactly all ghost terms in the Eagon-Northcott resolution (2.5) coming from $a_{j}=b_{i}$; in particular a resolution of $R / I_{g}$ of $W_{s}\left(\underline{b}_{\hat{i}} ; \underline{a}_{\hat{j}}\right)$ is given by its Eagon-Northcott resolution.

Proof. As observed in the proof of Lemma 2.1, the vanishing of $\operatorname{Ext}_{R}^{i}\left(R / I_{t}(\mathcal{A}), R\right)$ is an open property. This holds also for the elements $R / I_{t}(\mathcal{A})$ of $\operatorname{Grad} \operatorname{Alg}(H)$, i.e. the subset of $\operatorname{GradAlg}(H)$ such that $I_{t}(\mathcal{A})$ has maximal codimension in $R$ (this subset is $W_{s}(\underline{b} ; \underline{a})$ ) is open. Since it is irreducible by Lemma 2.1, we have proved the open irreducible property stated in Proposition [5.1, and it remains to see the inclusion $W_{s}\left(\underline{b}_{\hat{i}} ; \underline{a}_{\hat{j}}\right) \subset W_{s}(\underline{b} ; \underline{a})$ and the existence of generizations.

We claim that the elements $R / I_{t}(\mathcal{A})$ of $W_{s}(\underline{b} ; \underline{a})$ whose matrix $\mathcal{A}$ contains a unit of the field $k$ at the $(i, j)$-entry, belong to $W_{s}\left(\underline{b}_{\hat{i}} ; \underline{a}_{\hat{j}}\right)$. Indeed $\mathcal{A}$ is a presentation matrix of $M=\operatorname{coker} \varphi^{*}$ and by rearranging the direct summands of the source and target of the morphism $\varphi^{*}$, we may assume $(i, j)=(1,0)$. By elementary row operations we transform 
$\mathcal{A}$ to a matrix with only zeros (and one 1 ) in the first column, i.e. there is an invertible $t \times t$ matrix $\mathcal{C}$ such that the cokernel of the map induced by $\mathcal{C} \cdot \mathcal{A}$ is isomorphic to $M$. By Fitting's lemma, $I_{t}(\mathcal{A})=I_{t}(\mathcal{C} \cdot \mathcal{A})$. Moreover the $(t-1) \times(t+c-2)$ matrix $\mathcal{A}^{\prime}$ obtained by deleting the first row and column of $\mathcal{C} \cdot \mathcal{A}$ satisfies $I_{t-1}\left(\mathcal{A}^{\prime}\right)=I_{t}(\mathcal{C} \cdot \mathcal{A})$ and defines a determinantal ring of $W_{s}\left(\underline{b}_{\hat{i}} ; \underline{a}_{\hat{j}}\right)$, and we have proved the inclusion and the whole claim (but taking any $R / I_{t}\left(\mathcal{A}^{\prime}\right)$ of $W_{s}\left(\underline{b}_{\hat{i}} ; \underline{a}_{\hat{j}}\right)$ and putting $\mathcal{A}=\left(\begin{array}{cc}1 & 0 \\ 0 & \mathcal{A}^{\prime}\end{array}\right)$ we get $\left(R / I_{t}(\mathcal{A})\right) \in W_{s}(\underline{b} ; \underline{a})$ which is an easier argument for the inclusion).

Finally to see the existence of the generization of $R / I$ of $W_{s}(\underline{b} ; \underline{a}) \backslash W_{s}\left(\underline{b}_{\hat{i}} ; \underline{a}_{\hat{j}}\right), I=$ $I_{t}(\mathcal{A})$, we may assume that $\mathcal{A}$ has a 0 at the $(i, j)$-entry by the proven claim. Then we apply Lemma 2.8 to $T=k[u]_{\wp}, \wp=(u)$, letting $u=0$ correspond to $\mathcal{A}$ and $\mathcal{A}_{T}$ to a matrix obtained by replacing the 0 of the $(i, j)$-entry by $u$ and all other $\left(i^{\prime}, j^{\prime}\right)$-entries by polynomials of $R \otimes k[u]$ of degree $a_{j^{\prime}}-b_{i^{\prime}}$ with coefficients in $k[u]$ such that the choice $u=1$ makes the corresponding matrix general enough. More precisely, with notations as above, i.e. with $(i, j)=(1,0)$ etc., we may choose the coefficients of the polynomials of the 1 . column such that the choice $u=1$ make the entries equal to 0 for all $(i, 0)$ with $i>1$ and the entries of $\mathcal{A}^{\prime}$ general. In some open subset $U \ni\{0,1\}$ of $\operatorname{Spec}(k[u])$ the ideal $I_{t}\left(\mathcal{A}_{u}\right)$ of the matrices given by $\mathcal{A}_{u}$ for $u \in U \backslash\{0\}$ has maximal codimension in $R$ and we are done.

Corollary 5.2. Let $I=I_{t}(\mathcal{A}), R / I \in W_{s}(\underline{b} ; \underline{a})$, and suppose that the ideal of submaximal minors $I_{t-1}(\mathcal{A})=R$. Then $R / I$ is a complete intersection of $R$.

Proof. Suppose $I_{t-1}(\mathcal{A})=R$. Since the submaximal minors are homogeneous and generate $I_{t-1}(\mathcal{A})$, one of the submaximal minors must be a unit, whence at least one of the entries of $\mathcal{A}$ is a unit. Using the notations and the proven claim in the proof above, we have $I_{t}(\mathcal{A})=I_{t}(\mathcal{C} \cdot \mathcal{A})=I_{t-1}\left(\mathcal{A}^{\prime}\right)$, and we get $I_{t-1}(\mathcal{A})=I_{t-1}(\mathcal{C} \cdot \mathcal{A})=I_{t-2}\left(\mathcal{A}^{\prime}\right)$ for $t \geq 3$ by the same arguments. Since $I_{1}\left(\mathcal{A}^{\prime}\right)$ is a c.i. of $R$, we conclude by induction on $t$.

Remark 5.3. The ghost terms treated in Proposition 5.1 are removable under generization, while all other ghost terms appearing in the Eagon-Northcott resolution of $R / I_{t}(\mathcal{A})$ may sometimes probably be removed, but not always. Indeed the latter can not be removed under generization provided every graded deformation of $R / I_{t}(\mathcal{A})$ comes from deforming $\mathcal{A}$ because, in this case, $\overline{W_{s}(\underline{b} ; \underline{a})}$ is an irreducible component of $\operatorname{Grad} \operatorname{Alg}(H)$, whence the minimal resolution of its general element is given by some Eagon-Northcott resolution of $R / I_{t}\left(\mathcal{A}^{\prime}\right)$ with $\mathcal{A}^{\prime}$ minimal, cf. Example 5.5 below. Note that Theorem 4.6 and Theorem 4.8 give conditions under which every graded deformation of $R / I_{t}(\mathcal{A})$ comes from deforming $\mathcal{A}$. In particular if $\operatorname{dim} R / I_{t}(\mathcal{A}) \geq 3$ and $a_{i-\min (3, t)} \geq b_{i}$ for $\min (3, t) \leq i \leq t$, only ghost terms as in Proposition 5.1 are removable under generization!

Example 5.4. (removing all ghost terms using Proposition 5.1)

(i) Let $R=k\left[x_{0}, x_{1}\right]$, let $\mathcal{B}$ be a general $2 \times 3$ matrix with linear (resp. quadratic) entries in the first (resp. second) row and let $\mathcal{A}=\left(\begin{array}{cc}u & v \\ w & \mathcal{B}\end{array}\right)$ where $u \in k, v$ a row of general linear forms and $w$ a column whose transpose is $\left(0, x_{0}\right)$. The vanishing of all $3 \times 3$ minors defines an artinian ring with $h$-vector $(1,2,3,1)$ for every $u \in k$. If $I$ (resp. $I_{g}$ ) is the ideal given by all $3 \times 3$ minors of $\mathcal{A}$ with $u=0$ (resp. $u=1$ ), then $(R / I) \in W_{s}(-2,-1,-1 ;-1,0,0,0)$ and $\left(R / I_{g}\right) \in W_{s}(-2,-1 ; 0,0,0)$ and using Macaulay 2 we find minimal resolutions

$$
0 \rightarrow R(-5) \oplus R(-4)^{2} \rightarrow R(-4) \oplus R(-3)^{3} \rightarrow I \rightarrow 0,
$$




$$
0 \longrightarrow R(-5) \oplus R(-4) \longrightarrow R(-3)^{3} \longrightarrow I_{g} \longrightarrow 0
$$

Here $R / I_{g}$ is a generization of $R / I$ in $\operatorname{GradAlg}(H)$.

(ii) Let $R=k\left[x_{0}, x_{1}, x_{2}\right]$, let $\mathcal{B}$ be a general $2 \times 4$ matrix with linear (resp. cubic) entries in the first (resp. second) row and let $\mathcal{A}=\left(\begin{array}{cc}u & v \\ w & \mathcal{B}\end{array}\right)$ where $u \in k, v$ a row of general linear forms and $w$ the column whose transpose is $\left(0, x_{0}^{2}\right)$. Using Macaulay 2 we get that the vanishing of all $3 \times 3$ minors defines an artinian ring with $h$-vector $(1,3,6,10,9,7,3)$. If $I$ (resp. $I_{g}$ ) is the ideal given by the $3 \times 3$ minors of $\mathcal{A}$ with $u=0$ (resp. $u=1$ ), then $(R / I) \in W_{s}(-3,-1,-1 ;-1,0,0,0,0)$ and $\left(R / I_{g}\right) \in W_{s}(-3,-1 ; 0,0,0,0)$ and we have minimal resolutions

$$
\begin{gathered}
0 \rightarrow R(-10) \oplus R(-8) \oplus R(-6) \rightarrow R(-7)^{4} \oplus R(-5)^{4} \rightarrow R(-4)^{6} \rightarrow I_{g} \rightarrow 0, \\
0 \rightarrow R(-10) \oplus R(-8)^{2} \oplus R(-6)^{3} \rightarrow R(-8) \oplus R(-7)^{4} \oplus R(-6)^{2} \oplus R(-5)^{8} \rightarrow R(-5)^{4} \oplus R(-4)^{6} \rightarrow I .
\end{gathered}
$$

Example 5.5. (removing some ghost terms using Proposition 5.1)

Let $R=k\left[x_{0}, x_{1}, x_{2}\right]$ and let $\mathcal{A}=\left(\begin{array}{cc}u & v \\ w & \mathcal{B}\end{array}\right)$ be as in Example 5.4 (ii) only replacing cubic by quadratic and $w^{T}$ by $\left(0, x_{0}\right)$. Using Macaulay 2 we get that the vanishing of all $3 \times 3$ minors defines an artinian ring with $h$-vector $(1,3,6,4,1)$. If $I$ (resp. $\left.I_{g}\right)$ is the ideal generated by the $3 \times 3$ minors of $\mathcal{A}$ for $u=0$ (resp. $u=1$ ), then $(R / I) \in W_{s}(-2,-1,-1 ;-1,0,0,0,0)$ and $\left(R / I_{g}\right) \in W_{s}(-2,-1 ; 0,0,0,0)$ and we have minimal resolutions

$$
0 \rightarrow R(-7) \oplus R(-6)^{2} \oplus R(-5)^{3} \rightarrow R(-6) \oplus R(-5)^{6} \oplus R(-4)^{8} \rightarrow R(-4)^{4} \oplus R(-3)^{6} \rightarrow I \rightarrow 0 .
$$

Observe that there is still a ghost term in the resolution of $I_{g}$. In the artinian case, however, we have seen in Example 4.4 that $\overline{W_{s}(-2,-1 ; 0,0,0,0)}$ is not an irreducible component of $\operatorname{GradAlg}(H)$. So there is a possibility for removing this ghost under a generization to a non-determinantal artinian ring! Indeed M. Boij pointed out in a note he sent me that a general artinian algebra with Hilbert function $(1,3,6,4,1)$ can be seen as a type two algebra $A$ given by the inverse system $(f, g)$ where $\operatorname{deg} f=4$ and $\operatorname{deg} g=3$. Following Boij's note, this algebra has a minimal resolution as in (5.1) with the ghost term $R(-5)$ removed and $A$ is probably a generization of $R / I_{g}$.

On the other hand, we may very well consider the determinantal algebras $R / I$ and $R / I_{g}$ described above in the polynomial ring $R=k\left[x_{0}, x_{1}, \cdots, x_{n}\right]$ for $n \geq 5$. This leads to determinantal rings of dimension greater or equal to 3 with minimal resolutions exactly as in (5.1) and (5.2). Thanks to Theorem 4.6, see Remark 5.3, $\overline{W_{s}(-2,-1 ; 0,0,0,0)}$ is in this case an irreducible component of $\operatorname{GradAlg}(H)$ (as well as of $\operatorname{Hilb}^{p}\left(\mathbb{P}^{n}\right)$, up to closure), whence $R / I_{g}$ has no generization to non-determinantal rings and the ghost term $R(-5)$ of (5.1) remains a ghost term for the general element of the component.

Finally suppose $c=2$. Then repeated use of Proposition 5.1 will remove all ghost terms in the minimal resolution of $I$ since the transpose of $\mathcal{A}$ is the Hilbert-Burch matrix. This is rather well known (cf. [12, Thm. 2(iii)]); indeed even more precise results are true, see [20] which finds the codimension of all "ghost terms strata" when $\operatorname{dim} A$ is small and $A$ is Cohen-Macaulay. Note that any codimension-2 Cohen-Macaulay quotient $R / I$ is determinantal, so Proposition 5.1 applies to such $R / I$. If $A=R / I$ is the homogeneous coordinate ring of a codimension- 2 scheme $X$ in $\mathbb{P}^{n}$, then $A$ is Cohen-Macaulay for $\operatorname{dim} A=$ 
1 and there are no ghost terms in the minimal resolution of $I:=I_{X}$ for $A$ general. If $X$ is a locally Cohen-Macaulay (lCM) curve in $\mathbb{P}^{3}$ and $A$ is not necessarily Cohen-Macaulay, then we can generalize the removal of ghost terms above for codimension-2 quotients to the following.

Proposition 5.6. [30, Thm. 2.8] Let $X \subset \mathbb{P}^{3}$ be any $l C M$ curve and let $0 \rightarrow L_{4} \stackrel{\sigma}{\rightarrow} L_{3} \rightarrow$ $\cdots \rightarrow M \rightarrow 0$ be a minimal $R$-free resolution of the Rao module $M:=H_{*}^{1}\left(\mathcal{I}_{X}\right)$. By [47] there is a minimal $R$-free resolution of the following form

$$
0 \rightarrow L_{4} \stackrel{\sigma \oplus 0}{\longrightarrow} L_{3} \oplus P_{2} \rightarrow P_{1} \rightarrow I_{X} \rightarrow 0
$$

(i.e. the composition $L_{4} \stackrel{\sigma \oplus 0}{\longrightarrow} L_{3} \oplus P_{2} \rightarrow P_{2}$ is zero). If $P_{1}$ and $P_{2}$ have a common free summand; $P_{2}=P_{2}^{\prime} \oplus R(-i), P_{1}=P_{1}^{\prime} \oplus R(-i)$, then there is a generization $X^{\prime}$ of $X$ in $\operatorname{GradAlg}(H)$ with minimal resolution

$$
0 \rightarrow L_{4} \stackrel{\sigma \oplus 0}{\longrightarrow} L_{3} \oplus P_{2}^{\prime} \rightarrow P_{1}^{\prime} \rightarrow I_{X^{\prime}} \rightarrow 0 .
$$

Note that the statement "a generization $X^{\prime}$ of $X$ in $\operatorname{Hilb}^{p}\left(\mathbb{P}^{3}\right)$ with constant postulation" in [30, Thm. 2.8] really means "a generization $X^{\prime}$ of $X$ in $\operatorname{GradAlg}(H)$ ". This result applies to a codimension-2 Cohen-Macaulay quotient $R / I_{X}$ by letting $M=0$, which implies $L_{3}=L_{4}=0$, coinciding with Proposition 5.1 in this case.

\section{UPGRADING OF PREVIOUS RESUlts}

In this section we generalize main theorems from [29, 34] by using Theorems 4.1 and 4.6 for $\operatorname{dim} A \geq 1$, or more precisely we use that the two conjectures in [34 mentioned in the Introduction now are theorems (for $n-c>0$ ). Indeed the previous proofs relied on the part of the conjectures which was proved (in [33]) at the time these papers were written, but we can, using mainly the "same" proofs as in [29, 34], get stronger results (the same conclusions under weaker assumptions). To better understand the proofs presented here, it may be a good idea to consider the corresponding proofs in [29] simultaneously.

We start, however, with [34]. The following theorem was proved in [34, Thm. 3.2] under some assumptions (mainly " $a_{0}>b_{t}, a_{t+3}>a_{t-2}$ and $n-c \geq 0$ ") and it was in [31, Cor. 5.7] shown to be true in general for $n-c \geq 1$ (under elsewhere weaker assumptions: $W(\underline{b} ; \underline{a}) \neq \emptyset$ and $a_{i-2} \geq b_{i}$ for $i \geq 2$ ). For $n=c,[34$, Thm. 3.2] is still the best result we have with assumptions only on $a_{j}$ and $b_{i}$, and we can immediately generalize it to

Theorem 6.1. Let $X \subset \mathbb{P}^{n}$ be a general determinantal scheme of codimension $c$ and suppose $a_{0}>b_{t}$ and $a_{t+c-2}>a_{t-2}$. Then (cf. Remark 2.6)

$$
\operatorname{dim} W(\underline{b} ; \underline{a})=\lambda_{c}+K_{3}+K_{4}+\cdots+K_{c} .
$$

Proof. This follows from [34, Prop. 3.1], see Proposition 6.3(i) below, and [31, Cor. 5.7].

Now, to generalize the main results of [29], we first restate [29, Prop. 3.4]. With notations as in Sec. 2 (i.e. we delete the last column of $\mathcal{A}$ to get $\mathcal{A}_{c-1}$ and the map $\varphi_{c-1}$ induced by the transpose of $\left.\mathcal{A}_{c-1}\right)$, let $\mathcal{B}:=\mathcal{A}_{c-1}, B:=R / I_{t}(\mathcal{B})$ and $N:=$ coker $\varphi_{c-1}^{*}$. In this section we let $A:=R / I_{t}(\mathcal{A})$ be good determinantal, i.e. $(A) \in W(\underline{b} ; \underline{a})$ where $\underline{a}=a_{0}, a_{1}, \ldots, a_{t+c-2}$, and as previously we assume that all entries of the deleted column $(s)$ 
belong to $\mathfrak{m}$. It follows that $(B) \in W\left(\underline{b} ; \underline{a^{\prime}}\right)$ with $\underline{a^{\prime}}=a_{0}, a_{1}, \ldots, a_{t+c-3}$ by [2] and the inclusions $I_{t}(\mathcal{B}) \subset I_{t}(\mathcal{A}) \subset I_{t-1}(\mathcal{B})$. We set $X=\operatorname{Proj}(A)$ and $Y=\operatorname{Proj}(B)$.

Proposition 6.2. Let $c \geq 3$, let $(X) \in W(\underline{b} ; \underline{a})$ be general and suppose $\operatorname{dim} W\left(\underline{b} ; \underline{a^{\prime}}\right) \geq$ $\lambda_{c-1}+K_{3}+K_{4}+\ldots+K_{c-1}\left(\right.$ and $\geq \lambda_{2}$ if $c=3$ ). If

$$
{ }_{0} \operatorname{hom}_{R}\left(I_{Y}, I_{X / Y}\right) \leq \sum_{j=0}^{t+c-3}\left(\begin{array}{c}
a_{j}-a_{t+c-2}+n \\
n
\end{array}\right),
$$

then $\operatorname{dim} W(\underline{b} ; \underline{a})=\lambda_{c}+K_{3}+K_{4}+\ldots+K_{c}$. We also get equality in (6.1), as well as

$$
\operatorname{dim} W(\underline{b} ; \underline{a})=\operatorname{dim} W\left(\underline{b} ; \underline{a^{\prime}}\right)+\operatorname{dim}_{k} N\left(a_{t+c-2}\right)_{0}-1-{ }_{0} \operatorname{hom}_{R}\left(I_{Y}, I_{X / Y}\right) .
$$

See [29, Prop. 3.4] for a proof, and notice that since $\operatorname{dim} A \geq 1$ and $X$ is general in $W(\underline{b} ; \underline{a})$, the assumption $\operatorname{depth}_{I(Z)} B \geq 2$ of [29, Prop. 3.4] holds letting $Z:=V\left(I_{t-1}(\mathcal{B})\right)$.

Then [34, Prop. 3.1] is mainly (i) in the following.

Proposition 6.3. (i) If $a_{0}>b_{t}$ and $a_{t+c-2}>a_{t-2}$, then (6.1) holds for $X$ general.

(ii) If ${ }_{0} \operatorname{Ext}_{B}^{i}\left(N, B\left(-a_{t+c-2}\right)\right)=0$ for $i=1$ and 2 , then (6.1) holds. In particular (6.1) holds provided depth $\operatorname{It-1}_{t(\mathcal{B})} B \geq 3$ (and $\operatorname{Ext}_{B}^{1}(N, B)=0$ provided $\operatorname{depth}_{I_{t-1}(\mathcal{B})} B \geq 2$ ).

Proof. (ii) The arguments we apply in the proof are very similar to those needed in (3.1), (3.2) and (3.3) to get related statements, except that we now use the spectral sequence $E_{2}^{p, q}:=\operatorname{Ext}_{B}^{p}\left(\operatorname{Tor}_{q}^{R}(B, N), B\right) \Rightarrow \operatorname{Ext}_{R}^{p+q}(N, B)$ and its corresponding 5-term exact sequence. Indeed due to assumptions and (2.10) we get isomorphisms

$$
{ }_{v} \operatorname{Ext}_{R}^{1}(N, B) \simeq\left(E_{2}^{0,1}\right)_{v} \simeq{ }_{v} \operatorname{Hom}_{R}\left(I_{Y}, \operatorname{Hom}_{B}(N, B)\right) \simeq{ }_{v} \operatorname{Hom}_{R}\left(I_{Y}, I_{X / Y}\left(a_{t+c-2}\right)\right)
$$

for $v=-a_{t+c-2}$, Moreover as in (3.3), cf. (2.7), we get for this $v$ the exact sequence

$$
0 \rightarrow{ }_{v} \operatorname{Hom}_{R}(N, B) \rightarrow{ }_{v} \operatorname{Hom}_{R}\left(F^{*}, B\right) \rightarrow{ }_{v} \operatorname{Hom}_{R}\left(G_{c-1}^{*}, B\right) \rightarrow{ }_{v} \operatorname{Ext}_{R}^{1}(N, B) \rightarrow 0
$$

where ${ }_{v} \operatorname{Hom}_{R}\left(F^{*}, B\right) \simeq \oplus_{i=1}^{t} B_{\left(b_{i}+v\right)}=0$ and ${ }_{v} \operatorname{hom}_{R}\left(G_{c-1}^{*}, B\right) \simeq \operatorname{dim}\left(\oplus_{j=0}^{t+c-3} B_{\left(a_{j}+v\right)}\right)=$ $\sum_{j=0}^{t+c-3}\left(\begin{array}{c}a_{j}-a_{t+c-2}+n \\ n\end{array}\right)$. This implies (6.1) .

The above arguments are used in [35, Rem. 3.4] and the proof of [35, Prop. 3.5] from which we get (6.1) under the assumption $\operatorname{depth}_{I_{t-1}(\mathcal{B})} B \geq 4$. To see that we can weaken the depth assumption to $\operatorname{depth}_{I_{t-1}(\mathcal{B})} B \geq 3$, we really need to refine the argument. Following, however, the proof of [31, Thm. 4.5] exactly as in the paragraph after (4.3) in [31, we get $\operatorname{Ext}_{B}^{i}(N, B)=0$ for $i=1$ and 2 (resp. for $i=1$ ) under the assumption $\operatorname{depth}_{I_{t-1}(\mathcal{B})} B \geq 3$ (resp. $\operatorname{depth}_{I_{t-1}(\mathcal{B})} B \geq 2$ ), and we are done.

Remark 6.4. Using Propositions 6.2 and 6.3)(ii) one may reprove the dimension formula $\operatorname{dim} W(\underline{b} ; \underline{a})=\lambda_{c}+K_{3}+K_{4}+\cdots+K_{c}$ of Theorem 4.1 in the case $n-c \geq 1, a_{i-2} \geq b_{i}$ for $i \geq 2$ and $W(\underline{b} ; \underline{a}) \neq \emptyset$ by using the recursive strategy of successively deleting columns of $\mathcal{A}$ from the right-hand side, see Introduction and Remark 2.6. In [35] we pointed out that [35, Cor. 3.19] shows that the recursive strategy also applies to reprove the generic smoothness of $W(\underline{b} ; \underline{a})$ of Theorem 4.6] in the case $n-c \geq 2$ and $a_{0} \geq b_{t}$ (due to Remark 2.5. the latter assumption may here be weakened to $a_{i-\min (3, t)} \geq b_{i}$ for $\left.\min (3, t) \leq i \leq t\right)$. This means that we have two rather different proofs for the two conjectures of [34]. 
With the above propositions we start by considering determinantal curves. In this and later results we denote by $\tau_{X / Y}$ the following morphism induced by $I_{X / Y} \hookrightarrow B$ :

$$
\tau_{X / Y}:{ }_{0} \operatorname{Ext}_{B}^{1}\left(I_{Y} / I_{Y}^{2}, I_{X / Y}\right) \rightarrow{ }_{0} \operatorname{Ext}_{B}^{1}\left(I_{Y} / I_{Y}^{2}, B\right) .
$$

A key result of [29] (Prop. 4.6) shows that if $\operatorname{ker} \tau_{X / Y}=0, \operatorname{depth}_{I_{t-1}(\mathcal{B})} B \geq 3$ and $X$ good determinantal, then the property "every deformation of a determinantal scheme comes from deforming the matrix" is transferred from $Y$ to $X$. Recalling that $\operatorname{dim} W(\underline{b} ; \underline{a})=$ $\lambda_{c}+K_{3}+\ldots+K_{c}$ is now a theorem when $n-c=1$, the following result generalizes [29, Prop. 4.15] to arbitrary $c \geq 3$. (For $c=2$ we have a more complete result with stronger conclusions in Theorem 4.15).

Proposition 6.5. Let $X=\operatorname{Proj}(A) \subset \mathbb{P}^{n}, A=R / I_{t}(\mathcal{A})$, be general in $W(\underline{b} ; \underline{a})$ and suppose $a_{i-\min (3, t)} \geq b_{i}$ for $\min (3, t) \leq i \leq t, c \geq 3$ and $\operatorname{dim} X=n-c=1$. If $Y=\operatorname{Proj}(B)$ is defined by the vanishing of the maximal minors of the matrix obtained by deleting the last column of $\mathcal{A}$, then the following statements hold:

(i) If $\tau_{X / Y}:{ }_{0} \operatorname{Ext}_{B}^{1}\left(I_{Y} / I_{Y}^{2}, I_{X / Y}\right) \rightarrow{ }_{0} \operatorname{Ext}_{B}^{1}\left(I_{Y} / I_{Y}^{2}, B\right)$ is injective, then $X$ is unobstructed and $\overline{W(\underline{b} ; \underline{a})}$ is a generically smooth irreducible component of $\operatorname{Hilb}^{p}\left(\mathbb{P}^{n}\right)$. Moreover every deformation of $X$ comes from deforming $\mathcal{A}$.

(ii) If ${ }_{0} \operatorname{Ext}_{A}^{1}\left(I_{X} / I_{X}^{2}, A\right)=0$, then $X$ is unobstructed, and

$$
\operatorname{codim}_{\mathrm{Hilb}^{p}\left(\mathbb{P}^{n}\right)} \overline{W(\underline{b} ; \underline{a})}=\operatorname{dim} \operatorname{ker} \tau_{X / Y}-{ }_{0} \operatorname{ext}_{B}^{1}\left(I_{X / Y}, A\right) .
$$

(iii) We always have

$$
\operatorname{codim}_{\mathrm{Hilb}^{p}\left(\mathbb{P}^{n}\right)} \overline{W(\underline{b} ; \underline{a})} \leq \operatorname{dim} \operatorname{ker} \tau_{X / Y}
$$

Moreover if ${ }_{0} \operatorname{Ext}_{B}^{1}\left(I_{X / Y}, A\right)=0$, then we have equality in (6.3) if and only if $X$ is unobstructed.

Proof. Thanks to Proposition 6.3(ii) the proof is the "same" as for [29, Prop. 4.15]. Indeed for (i) we apply Theorem 4.6 onto $W\left(\underline{b} ; \underline{a^{\prime}}\right) \ni(Y)$ instead of the corresponding result of [29] which required $c-1 \leq 4$ and we get (i) from [29, Thm. 4.6] and Lemma 2.10. For (ii) and (iii) we need the final dimension formula of Proposition 6.2 to use the proof of [29]. Using Proposition 6.3(ii) we get the mentioned dimension formula and we are done.

We consider now zero dimensional determinantal schemes $(n-c=0)$. In this case we also need to consider the morphism

$$
\rho^{1}:=\rho_{X / Y}^{1}:{ }_{0} \operatorname{Ext}_{B}^{1}\left(I_{X / Y}, I_{X / Y}\right) \rightarrow{ }_{0} \operatorname{Ext}_{B}^{1}\left(I_{X / Y}, B\right)
$$

induced by $I_{X / Y} \hookrightarrow B$. In addition to [29, Prop. 4.6] which states that $\operatorname{ker} \tau_{X / Y}=0$, ker $\rho^{1}=0$ and $\operatorname{depth}_{I_{t-1}(\mathcal{B})} B=2$ transfer the property "every deformation of a determinantal ring comes from deforming the matrix" from $B$ (or $Y$ ) to $A$, we need a variation of [29, Prop. 4.6] which don't require ker $\rho^{1}=0$ (see [29, Prop. 4.13] for the details). Below we generalize [29, Thm. 4.19] by replacing the assumption $4 \leq c \leq 6$ in [29, Thm. 4.19] by $c \geq 4$. Note that we denote $\operatorname{GradAlg}(H)$ by $\operatorname{Hilb}^{H}\left(\mathbb{P}^{n}\right)$ since $\operatorname{dim} A=1$, cf. the text before (2.1) and see Remark 2.6 for computing $\operatorname{dim} W(\underline{b} ; \underline{a})$. We refer to [29] for examples. 
Theorem 6.6. Let $X=\operatorname{Proj}(A) \subset \mathbb{P}^{n}, A=R / I_{t}(\mathcal{A})$, be general in $W(\underline{b} ; \underline{a})$ and let $Y=\operatorname{Proj}(B)$ and $V=\operatorname{Proj}(C)$ be defined by the vanishing of the maximal minors of $\mathcal{B}$ and $\mathcal{C}$ respectively where $\mathcal{B}$ (resp. $\mathcal{C}$ ) is obtained by deleting the last column of $\mathcal{A}$ (resp. B). Suppose $\operatorname{dim} X=n-c=0, a_{i-\min (3, t)} \geq b_{i}$ for $\min (3, t) \leq i \leq t$ and suppose that (6.1) (cf. Proposition 6.3) holds. Moreover suppose

$$
\text { either } c=3 \text { or }\left[c \geq 4 \text { and } \operatorname{ker} \tau_{Y / V}=0\right] \text {. }
$$

Then $\operatorname{dim} W(\underline{b} ; \underline{a})=\lambda_{c}+K_{3}+\ldots+K_{c}$ and the following statements are true:

(i) If both $\rho^{1}:{ }_{0} \operatorname{Ext}_{B}^{1}\left(I_{X / Y}, I_{X / Y}\right) \rightarrow{ }_{0} \operatorname{Ext}_{B}^{1}\left(I_{X / Y}, B\right)$ and $\tau_{X / Y}:{ }_{0} \operatorname{Ext}_{B}^{1}\left(I_{Y} / I_{Y}^{2}, I_{X / Y}\right) \rightarrow$ ${ }_{0} \operatorname{Ext}_{B}^{1}\left(I_{Y} / I_{Y}^{2}, B\right)$ are injective, then $A$ is unobstructed and $\overline{W(\underline{b} ; \underline{a})}$ is a generically smooth irreducible component of $\mathrm{Hilb}^{H}\left(\mathbb{P}^{n}\right)$. Moreover every deformation of $A$ comes from deforming $\mathcal{A}$.

(ii) If ${ }_{0} \operatorname{Ext}_{B}^{1}\left(I_{X / Y}, A\right)=0$ and $\operatorname{ker} \tau_{X / Y}=0$, then $\overline{W(\underline{b} ; \underline{a})}$ belongs to a unique generically smooth irreducible component $Q$ of $\operatorname{Hilb}^{H}\left(\mathbb{P}^{n}\right)$ and the codimension of $\overline{W(\underline{b} ; \underline{a})}$ in $\operatorname{Hilb}^{H}\left(\mathbb{P}^{n}\right)$ is $\operatorname{dim} \operatorname{ker} \rho^{1}$. Indeed $A$ is unobstructed and

$$
\operatorname{dim} Q=\lambda_{c}+K_{3}+\ldots+K_{c}+\operatorname{dim} \operatorname{ker} \rho^{1} .
$$

(iii) If ${ }_{0} \operatorname{Ext}_{A}^{1}\left(I_{X} / I_{X}^{2}, A\right)=0$, then $A$ is unobstructed and

$$
\operatorname{codim}_{\mathrm{Hilb}^{H}\left(\mathbb{P}^{n}\right)} \overline{W(\underline{b} ; \underline{a})}=\operatorname{dim} \operatorname{ker} \rho^{1}+\operatorname{dim} \operatorname{ker} \tau_{X / Y}-{ }_{0} \operatorname{ext}_{B}^{1}\left(I_{X / Y}, A\right) .
$$

(iv) We always have $\operatorname{codim}_{\operatorname{Hilb}^{H}\left(\mathbb{P}^{n}\right)} \overline{W(\underline{b} ; \underline{a})} \leq \operatorname{dim} \operatorname{ker} \rho^{1}+\operatorname{dim} \operatorname{ker} \tau_{X / Y}$.

Suppose ${ }_{0} \operatorname{Ext}_{B}^{1}\left(I_{X / Y}, A\right)=0$. Then we have

$$
\operatorname{codim}_{\mathrm{Hilb}^{H}\left(\mathbb{P}^{n}\right)} \overline{W(\underline{b} ; \underline{a})}=\operatorname{dim} \operatorname{ker} \rho^{1}+\operatorname{dim} \operatorname{ker} \tau_{X / Y}
$$

if and only if $A$ is unobstructed.

Proof. Using Proposition 6.2 we get $\operatorname{dim} W(\underline{b} ; \underline{a})=\lambda_{c}+K_{3}+\ldots+K_{c}$ because Theorem 4.1 applies to $(B) \in W\left(\underline{b} ; \underline{a^{\prime}}\right)$ to find $\operatorname{dim} W\left(\underline{b} ; \underline{a^{\prime}}\right)$ where $\underline{a^{\prime}}=a_{0}, a_{1}, \ldots, a_{t+c-3}$. Then, due to [29, Prop. 4.6 and Prop. 4.13] the proof of the theorem is "the same" as the proof of [29, Thm. 4.19] since we already have generalized [29, Prop. 4.15] to Proposition 6.5] of this paper to cover determinantal curves $Y$ of arbitrary codimension $c \geq 3$. Note also that we have to delete a column of $\mathcal{B}$ (thus defining $V$ ) when we apply Proposition 6.5 to $Y$.

Remark 6.7. Looking at the proofs we see that we don't need to suppose (6.1) to get (the part of the) conclusions of (i) and (ii) that don't involve dimension and codimension formulas. Moreover note the overlap in (ii) and (iv) of the theorem.

Using again the variation [29, Prop. 4.13] we generalize [29, Prop. 4.24] to the following.

Proposition 6.8. With notations as in the first sentence of Theorem 6.6, suppose $c \geq 4$, $\operatorname{dim} X=n-c=0$ and $X$ general, $a_{i-\min (3, t)} \geq b_{i}$ for $\min (3, t) \leq i \leq t$ and suppose that (6.1) holds. Then $\operatorname{dim} W(\underline{b} ; \underline{a})=\lambda_{c}+K_{3}+\ldots+K_{c}$ (cf. Remark [2.6), and the following statements are true:

(i) If ${ }_{0} \operatorname{Ext}_{B}^{1}\left(I_{X / Y}, A\right)=0,{ }_{0} \operatorname{Ext}_{B}^{1}\left(I_{Y} / I_{Y}^{2}, I_{X / Y}\right)=0$ and ${ }_{0} \operatorname{Ext}_{B}^{1}\left(I_{Y} / I_{Y}^{2}, B\right)=0$ then $A$ is unobstructed. Moreover $W(\underline{b} ; \underline{a})$ is contained in a unique generically smooth irreducible component of $\operatorname{Hilb}^{H}\left(\mathbb{P}^{c}\right)$ of codimension $\operatorname{dim} \operatorname{ker} \rho_{X / Y}^{1}+\operatorname{dim} \operatorname{ker} \tau_{Y / V}-{ }_{0} \operatorname{ext}_{C}^{1}\left(I_{Y / V}, B\right)$. 


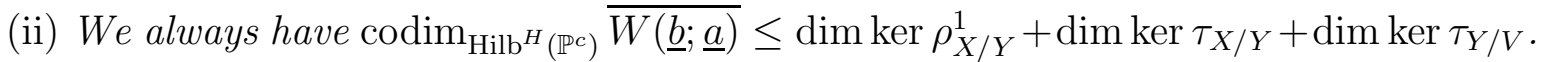
Suppose ${ }_{0} \operatorname{Ext}_{B}^{1}\left(I_{X / Y}, A\right)=0$ and ${ }_{0} \operatorname{Ext}_{C}^{1}\left(I_{Y / V}, B\right)=0$. Then we have

$$
\operatorname{codim}_{\mathrm{Hilb}^{H}\left(\mathbb{P}^{c}\right)} \overline{W(\underline{b} ; \underline{a})}=\operatorname{dim} \operatorname{ker} \rho_{X / Y}^{1}+\operatorname{dim} \operatorname{ker} \tau_{X / Y}+\operatorname{dim} \operatorname{ker} \tau_{Y / V}
$$

if and only if $A$ is unobstructed (e.g. $\left.{ }_{0} \operatorname{Ext}_{A}^{1}\left(I_{X} / I_{X}^{2}, A\right)=0\right)$.

Proof. Again we have $\operatorname{dim} W(\underline{b} ; \underline{a})=\lambda_{c}+K_{3}+\ldots+K_{c}$ by Proposition 6.2. Since Proposition 6.5 applies for every $c \geq 3$ we can use the proof of [29, Prop. 4.24] to get Proposition 6.8. Indeed we use Proposition 6.5(ii) and [29, Prop. 4.13] to get (i) above while we use the proof of Proposition 6.5(iii) to get (ii) (see [29] for details).

Acknowledgement. I thank prof. R.M. Miró-Roig at Barcelona for interesting discussions on this topic, prof. A. Iarrobino at Northeastern University, Boston and prof. M. Boij at KTH, Stockholm for comments and helping me approach the artinian case. Indeed this study of families of artinian determinantal rings started some time ago during a visit by A. Iarrobino and M. Boij at Oslo University College where we concretely studied examples of such families. I also thank the referee for some valuable comments.

\section{REFERENCES}

[1] M. André. Méthode Simpliciale en Algèbre Homologique et Algèbre Commutative. Lectures Notes in Math., no. 32, Springer-Verlag, New York, 1967.

[2] W. Bruns, The Eisenbud-Evans generalized principal ideal theorem and determinantal ideals, Proc. Amer. Math. Soc. 83 (1981), 19-24.

[3] W. Bruns and J. Herzog, Cohen-Macaulay rings, Cambridge Studies in Advanced Mathematics, 39. Cambridge University Press, Cambridge, 1993.

[4] W. Bruns and U. Vetter, Determinantal rings, Lect. Notes in Math., Vol. 1327, Springer-Verlag, New York/Berlin, 1988.

[5] D.A. Buchsbaum and D. Eisenbud, What Annihilates a Module? J. Algebra 47, (1977), 231-243.

[6] D.A. Buchsbaum and D.S. Rim, A generalized Koszul complex, II. Depth and multiplicity, Trans. Amer. Math. Soc., 111 (1964), 197-224.

[7] M.C. Chang, A filtered Bertini-type theorem, Crelle J. 397 (1989), 214-219.

[8] A. Conca, G. Valla Hilbert function of powers of ideals of low codimension Math. Z. 230 (1999), 753784 .

[9] J.A. Eagon and D.G. Northcott, Ideals defined by matrices and a certain complex associated with them, Proc. Roy. Soc. London 269 (1962), 188-204.

[10] D. Eisenbud, Commutative Algebra. With a view toward algebraic geometry, Springer-Verlag, Graduate Texts in Mathematics 150 (1995).

[11] D. Eisenbud, R. Hartshorne and F.-O. Schreyer, Twenty Points in $\mathbb{P}^{3}$, in: Recent Advances in Algebraic Geometry, London Math. Soc., Lect. Note Ser. 417, Cambridge University Press, 2015, 180-199.

[12] G. Ellingsrud, Sur le schéma de Hilbert des variétés de codimension 2 dans $\mathbb{P}^{e}$ a cône de CohenMacaulay, Ann. Scient. Éc. Norm. Sup. 8 (1975), 423-432.

[13] D. Faenzi and M.L. Fania, On the Hilbert scheme of determinantal subvarities, Math. Res. Lett. 21 no. 2, 297-311.

[14] G. Gotzmann. A Stratification of the Hilbert Scheme of Points in the Projective Plane, Math. Z. 199 no. 4 (1988) 539-547.

[15] D. Grayson and M. Stillman, Macaulay 2-a software system for algebraic geometry and commutative algebra, available at http://www.math.uiuc.edu/Macaulay2/ .

[16] A. Grothendieck, Les schémas de Hilbert, Séminaire N. Bourbaki, exp. 221 (1960/61), 249-276. 
[17] M. Haiman and B. Sturmfels, Multigraded Hilbert Schemes. J. Algebraic Geom. 13 (2004), 725-769.

[18] R. Hartshorne. Deformation theories. Graduate Texts in Math. Vol. 257 Springer-Verlag, New York, 2010.

[19] A. Iarrobino. Punctual Hilbert Schemes. Mem. Amer. Math. Soc. Vol. 10, no 188, (1977).

[20] A. Iarrobino. Betti strata of height two ideals J. Algebra 285 (2005), 835-855.

[21] A. Iarrobino and V. Kanev. Power sums, Gorenstein Algebras and Determinantal Loci. Lect. Notes in Math., Vol. 1721 Springer-Verlag, New York, 1999.

[22] R. Ile, Obstructions to deforming modules. PhD thesis, University of Oslo, 2001.

[23] R. Ile, Change of rings in Deformation theory of modules, Trans Amer. Math. Soc. 356 no. 12 (2004), 4873-4896.

[24] R. Ile, Deformation theory of rank one maximal Cohen-Macaulay modules on hypersurface singularities and the Scandinavian complex, Compos. Math. 140 (2004), 435-446.

[25] J.O. Kleppe. Deformations of graded algebras. Math. Scand. 45 (1979), 205-231.

[26] J.O. Kleppe, The smoothness and the dimension of $\mathrm{PGor}(H)$ and of other strata of the punctual Hilbert scheme. J. Algebra, 200 no. 2 (1998), 606-628.

[27] J.O. Kleppe, Maximal families of Gorenstein algebras. Trans. Amer. Math. Soc. 358 no. 7 (2006), 3133-3167.

[28] J.O. Kleppe, Families of Artinian and one-dimensional algebras. J. Algebra 311 (2007), 665-701

[29] J.O. Kleppe, Families of low dimensional determinantal schemes, J. Pure Appl. Algebra, 215 no. 7 (2011), 1711-1725. doi 10.1016/j.jpaa.2010.10.007

[30] J.O. Kleppe. The Hilbert Scheme of Buchsbaum Space Curves, Annales de l'institut Fourier 62 no. 6 (2012), p. 2099-2130.

[31] J.O. Kleppe, Deformations of modules of maximal grade and the Hilbert scheme at determinantal schemes., J. Algebra 338, (2014), 776-800.

[32] J.O. Kleppe, J. Migliore, R.M. Miró-Roig, U. Nagel and C. Peterson, Gorenstein liaison, complete intersection liaison invariants and unobstructedness, Memoirs A.M.S 732, (2001).

[33] J.O. Kleppe and R.M. Miró-Roig, Dimension of families of determinantal schemes, Trans. Amer. Math. Soc. 357, (2005), 2871-2907.

[34] J.O. Kleppe and R.M. Miró-Roig, Families of determinantal schemes, Proc. Amer. Math. Soc. 139 (2011), 3831-3843.

[35] J.O. Kleppe and R.M. Miró-Roig, On the normal sheaf of determinantal varieties J. Reine Angew. Math. http://dx.doi.org/10.1515/crelle-2014-0041. Online 18.06.2014.

[36] J. Kreuzer, J. Migliore, U. Nagel and C. Peterson, Determinantal schemes and Buchsbaum-Rim sheaves, J. Pure Appl. Algebra, 150 (2000), 155-174.

[37] D. Laksov, Deformation and transversality, Algebraic Geometry (Proc. Summer Meeting, Univ. Copenhagen, Copenhagen 1978, ed. K. Lønsted), Lect. Notes in Math., Springer-Verlag 732 (1979), 300-316.

[38] D. Laksov, Deformation of determinantal schemes, Compos. Math. 30 (1975), no. 3, 273-292.

[39] O.A. Laudal, Formal Moduli of Algebraic Structures. Lect. Notes in Math., Vol. 754, SpringerVerlag, New York, 1979.

[40] O.A. Laudal, Matrix Massey products and formal Moduli I, in: Algebra, Algebraic Topology and Their Interactions, Lect. Notes in Math., Vol. 1183, Springer-Verlag, New York, 1986, 218-240.

[41] A. Lorenzini, The minimal resolution conjecture, J. Algebra, 156 (1993), no. 1, 5-35.

[42] J. Migliore and M. Patnott, Minimal free resolutions of general points lying on cubic surfaces in $\mathbb{P}^{3}$, J. Pure Appl. Algebra, 215 no. 7 (2011) 1737-1746.

[43] J. Migliore, R.M. Miró-Roig and U. Nagel, Minimal resolutions of relatively compressed level algebras, J. Algebra, 284 (2005), no. 1, 333-370.

[44] R.M. Miró-Roig, Determinantal ideals, Birkhaüser, Progress in Math. 264, (2007).

[45] R.M. Miró-Roig and J. Pons-Llopis Minimal free resolution for points on surfaces, J. Algebra, 357 (2012), 304-318.

[46] M. Mustată Graded Betti Numbers of General Finite Subsets of Points on Projective Varieties, in: Suppl. Pragmatic, Le Mathematiche 53 (1998), 53-81. 
[47] A. P. Rao. Liaison Among Curves, Invent. Math. 50 (1979), 205-217.

[48] M. Schlessinger, Functors of Artin rings. Trans. Amer. Math. Soc. 130, (1968), 208-222.

[49] A. Siqveland, Generalized Matrix Massey Products for Graded Modules. J. Gen. Lie Theory Appl., Vol. 5 (2011), Article ID G110108, 15p., doi:10.4303/jglta/G110108.

[50] B. Ulrich, Ring of invariants and linkage of determinantal ideals, Math. Ann. 274 (1986), 1-17.

[51] C. Walter, Transversality theorems in general characteristic and arithmetically Buchsbaum schemes, Internat. J. Math. 5 (1994), no. 4, 609-617.

Faculty of Technology, Art and Design, Oslo and Akershus University College of Applied Sciences, P.O. Box 4, St. Olavs Plass, NO-0130 Oslo, Norway

E-mail address: JanOddvar.Kleppe@hioa.no 